\title{
Hepatic tristetraprolin promotes insulin resistance through RNA destabilization of FGF21
}

\author{
Konrad T. Sawicki, ${ }^{1}$ Hsiang-Chun Chang, ${ }^{1}$ Jason S. Shapiro, ${ }^{1}$ Marina Bayeva, ${ }^{1}$ Adam De Jesus, ${ }^{1}$ \\ Brian N. Finck, ${ }^{2}$ Jason A. Wertheim, ${ }^{3}$ Perry J. Blackshear, ${ }^{4}$ and Hossein Ardehali ${ }^{1}$ \\ ${ }^{1}$ Feinberg Cardiovascular Research Institute (FCVRI), Northwestern University, Chicago, Illinois, USA. ${ }^{2}$ Geriatrics and \\ Nutritional Sciences, Washington University in St. Louis, St. Louis, Missouri, USA. ${ }^{3}$ Department of Surgery, Northwestern \\ University, Chicago, Illinois, USA. ${ }^{4}$ Laboratory of Signal Transduction, National Institute of Environmental Health Sciences, \\ Research Triangle Park, North Carolina, USA.
}

\begin{abstract}
The role of posttranscriptional metabolic gene regulatory programs in diabetes is not well understood. Here, we show that the RNA-binding protein tristetraprolin (TTP) is reduced in the livers of diabetic mice and humans and is transcriptionally induced in response to insulin treatment in murine livers in vitro and in vivo. Liver-specific Ttp-KO (IsTtp-KO) mice challenged with highfat diet (HFD) have improved glucose tolerance and peripheral insulin sensitivity compared with littermate controls. Analysis of secreted hepatic factors demonstrated that fibroblast growth factor 21 (FGF21) is posttranscriptionally repressed by TTP. Consistent with increased FGF21, IsTtp-KO mice fed HFD have increased brown fat activation, peripheral tissue glucose uptake, and adiponectin production compared with littermate controls. Downregulation of hepatic Fgf21 via an adeno-associated virus-driven shRNA in mice fed HFD reverses the insulin-sensitizing effects of hepatic Ttp deletion. Thus, hepatic TTP posttranscriptionally regulates systemic insulin sensitivity in diabetes through liver-derived FGF21.
\end{abstract}

Authorship note: KTS, HCC, and JSS contributed equally to this work

Conflict of interest: The authors have declared that no conflict of interest exists.

Submitted: June 26, 2017

Accepted: May 23, 2018

Published: July 12, 2018

Reference information: JCI Insight. 2018;3(13):e95948. https://doi.org/10.1172/jci. insight. 95948.

\section{Introduction}

Eukaryotes have evolved a complex metabolic system that allows them to rapidly and appropriately respond to changes in nutritional status. The liver plays a major role in energy metabolism and is essential for the catabolism, anabolism, and redistribution of carbohydrates, fatty acids (FA), and amino acids. Hepatic energy metabolism is dictated by nutritional, hormonal, and neuronal signals leading to the expression of regulatory molecules, such as fibroblast growth factor 21 (FGF21) (1-3).

FGF21 is a hormone abundantly expressed in the liver, whose synthesis and secretion are highly responsive to nutrient intake in mice and humans. Hepatic FGF21 plays a key role in glucose and lipid metabolism, insulin sensitivity, and ketogenesis at both the hepatic and systemic levels. Liver-derived FGF21 acts on adipocytes to stimulate insulin-dependent glucose uptake, modulate lipolysis, and increase mitochondrial oxidative capacity (4-6). Administration of FGF21 to obese and diabetic mouse models stimulates the uptake of glucose in adipose tissue (AT) and improves systemic insulin sensitivity (7).

The coordination of these hepatic regulatory factors occurs on several levels to ensure an appropriate systemic metabolic response (8), although little is known about the regulation that occurs at the posttranscriptional level by RNA-binding proteins. Tristetraprolin (TTP, also known as ZFP36) is a Cys-Cys-Cys-His $(\mathrm{CCCH})$ tandem zinc finger (TZF) protein that posttranscriptionally represses gene expression by interacting with AU-rich elements (AREs) in the 3'-UTR of target mRNAs (9). After binding to the ARE of a target transcript, TTP promotes mRNA deadenylation and subsequent transcript decay (10). TTP has been well studied in the field of inflammation, where TNF- $\alpha$ is an established target of TTP (11). This is reflective of wholebody Ttp-KO mice, which develop a severe systemic autoimmune inflammatory syndrome shortly after birth due to elevated TNF- $\alpha$ accumulation in macrophages (12). TTP has also been implicated as a tumor suppressor gene, and we have shown that TTP is a critical regulator of cellular iron homeostasis $(13,14)$.

Recent reports suggest a potentially novel role for TTP in the regulation of metabolism. Ttp mRNA expression is responsive to insulin in cultured mouse fibroblasts and adipocytes, and a genetic linkage 
analysis identified TTP as a candidate gene for metabolic syndrome in humans (15-17). Studies in human subjects also revealed differences in TTP gene expression levels in the AT of obese men and women with metabolic syndrome compared with healthy obese men and women $(15,18)$. Although metabolic syndrome is a proinflammatory state, no correlation was observed between TTP expression and circulating TNF- $\alpha$ levels in humans, suggesting that the antiinflammatory effects of TTP are not responsible for its metabolic effects $(18,19)$. Studies in yeast also point toward the existence of a metabolic regulatory pathway involving yeast TTP family members. CTH1/2 are TZF proteins with sequence similarity to TTP and also function by binding to AREs in the 3'-UTR of their target mRNAs and by promoting mRNA transcript degradation. It has been shown that CTH1/2 degrade several mRNAs involved in metabolic processes $(20$, 21). However, the regulation of metabolic pathways by TTP in higher eukaryotes and the effects of insulin on these pathways through TTP are not known.

Here, we show that TTP is induced in the liver by insulin in vitro and in vivo and that TTP levels are reduced in the livers of diabetic mice and humans. To determine the effect of TTP on hepatic metabolism independent of inflammation, we generated liver-specific Ttp-KO (lsTtp-KO) mice. Loss of hepatic Ttp leads to improved systemic glucose tolerance and insulin sensitivity in mice fed high-fat diet (HFD). We show that TTP binds to and degrades the mRNA of FGF21, such that loss of hepatic Ttp amplifies the expression of Fgf21. Downregulation of hepatic Fgf21 expression in 1sTtp-KO mice reverses the protective effects against HFD. Thus, hepatic TTP is induced by insulin and modulates hepatic and systemic metabolism through posttranscriptional regulation of FGF21.

\section{Results}

Hepatic TTP levels are reduced in murine and human diabetes. Since the liver plays a critical role in systemic energy metabolism, we examined the levels of hepatic Ttp expression in diet-induced (HFD) and genetic $(d b / d b)$ murine models of diabetes. C57BL/6 mice fed $60 \%$ HFD for 8 weeks displayed reduced hepatic Ttp gene expression (Figure 1A) compared with mice fed a normal chow diet. In addition, 8-week-old $d b / d b$ mice exhibited reduced hepatic Ttp gene expression (Figure 1B) compared with nondiabetic control $(d b /+)$ mice. These results suggest that Ttp expression is reduced in the livers of diabetic mice via a leptin-independent mechanism. Similar to our results in mice, hepatic TTP mRNA expression was reduced in diabetic compared with nondiabetic human patients (Figure 1C and Supplemental Table 1; supplemental material available online with this article; https://doi.org/10.1172/ jci.insight.95948DS1).

Hepatic TTP levels are transcriptionally induced by insulin in vitro and in vivo. Ttp mRNA was previously shown to be induced by insulin in murine fibroblasts and adipocytes $(16,17)$. We subjected WT (Alb-Cre; $\left.T t p^{f / f}\right)$ primary mouse hepatocytes to insulin and found that hepatic Ttp mRNA is also induced by insulin in a time- and dose-dependent manner (Supplemental Figure 1, A and B), resulting in increased TTP protein levels (Supplemental Figure 1C). Additionally, i.p. injection of insulin in fasted WT mice also induced hepatic TTP at the protein and mRNA levels (Figure 1, D and E). The induction of Ttp by insulin was not due to a change in Ttp mRNA stability or synthesis of protein cofactors in primary hepatocytes, since insulin did not alter Ttp mRNA stability after actinomycin D treatment and cyclohexamide pretreatment did not prevent insulin-mediated Ttp induction (Supplemental Figure 1, D and E). Deletion studies of the murine Ttp promoter in WT mouse embryonic fibroblasts (MEFs) demonstrated that the proximal $137 \mathrm{bp}$ of the Ttp promoter were sufficient for full Ttp induction by insulin (Supplemental Figure 1F). Furthermore, the AKT and MAP kinase, but not protein kinase $\mathrm{C}$, pathways are involved in the Ttp induction by insulin in cultured MEFs (Supplemental Figure 1G). These results demonstrate that hepatic Ttp is transcriptionally induced by insulin in vitro and in vivo.

To determine if chronic hepatic insulin resistance is responsible for the reduction in hepatic TTP levels seen in diabetes, we subjected male $d b / d b$ and $d b /+$ mice to a bolus of i.p. insulin or saline and measured hepatic Ttp expression. While $d b /+$ mice exhibited a robust increase in hepatic TTP protein levels after insulin compared with saline injection, $d b / d b$ mice failed to demonstrate a similar effect (Figure $1 \mathrm{~F}$ ). Hepatic TTP protein levels after saline bolus were also higher in $d b /+$ mice compared with $d b / d b$ mice (Figure $1 \mathrm{~F}$ ), consistent with the reduced hepatic TTP baseline expression in diabetes. These results show that insulin resistance leads to impaired insulin-stimulated hepatic induction of TTP and suggest that chronic insulin resistance may contribute to the reduced basal levels of hepatic TTP in diabetic compared with nondiabetic mammals. 


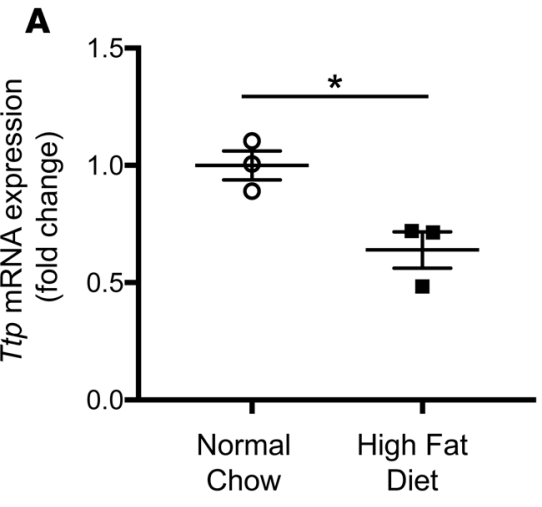

D

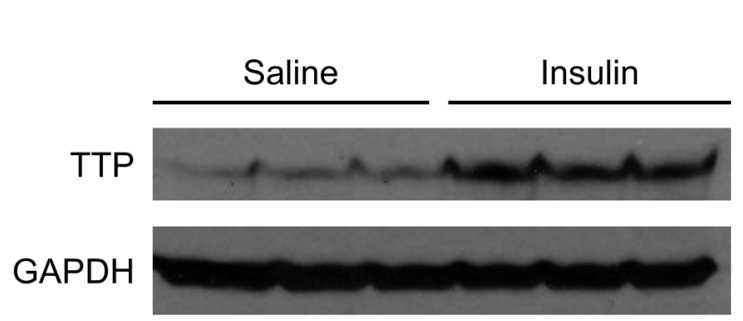

$\boldsymbol{F}$

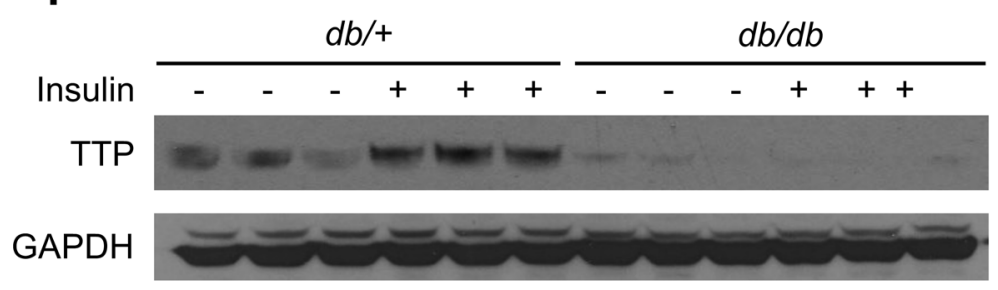

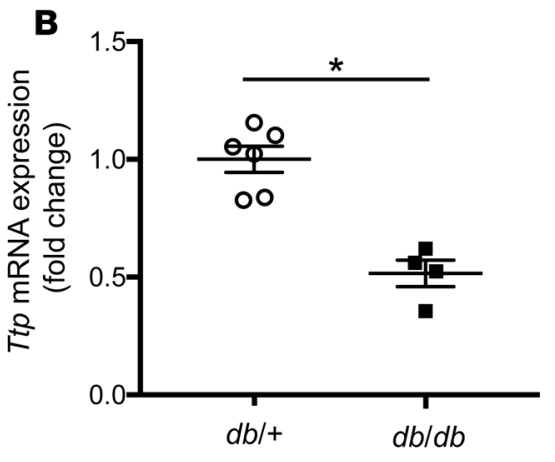

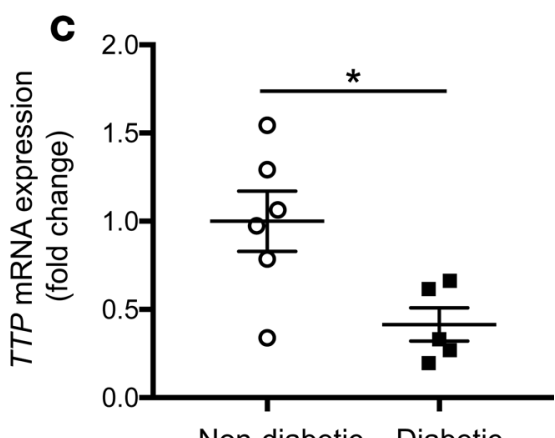

E
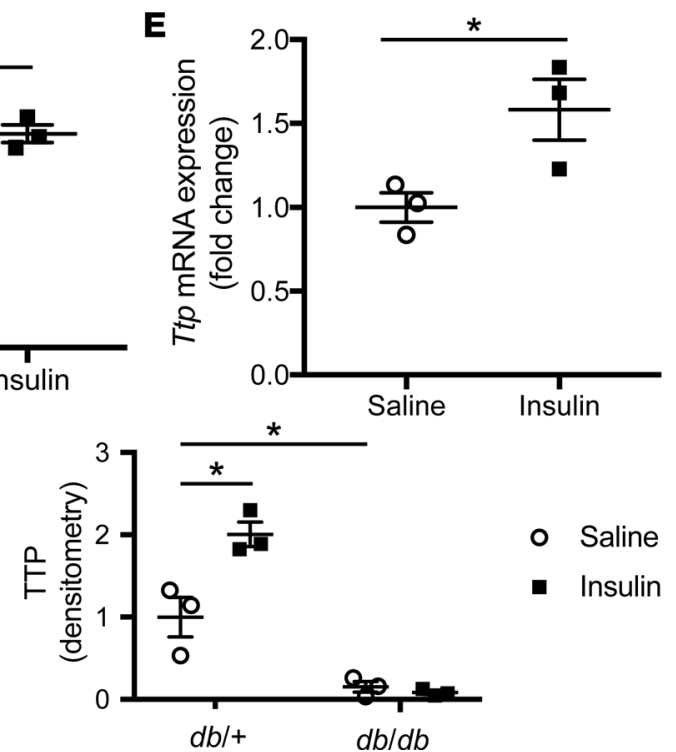

- Saline

- Insulin

Figure 1. Hepatic TTP is induced by insulin and reduced in diet-induced and genetic murine models of obesity and human diabetics. (A) Hepatic Ttp mRNA expression is reduced in C57BL/6 mice after 8 weeks of high-fat diet compared with normal chow $(n=4)$. (B) Hepatic Ttp mRNA expression is reduced in 8-week-old $d b / d b$ mice compared with $d b /+$ mice $(n=4)$. (C) Hepatic TTP mRNA expression is reduced in diabetic humans compared with nondiabetic humans $(n=6)$. (D) Hepatic TTP protein expression is induced in C57BL/6 mice after 4 hours of i.p. insulin injection compared with control saline injection $(n=3)$. Densitometry analysis is provided next to the Western blot. (E) Hepatic Ttp mRNA expression is induced in C57BL/ 6 mice after 4 hours of i.p. insulin injection compared with control saline injection $(n=3)$. (F) Hepatic protein levels of TTP are induced in 8-week-old $d b /+$ mice 4 hours after i.p. injection of insulin ( $0.0075 \mathrm{U} / \mathrm{g}$ body weight) compared with saline injection but not in $d b / d b$ mice $(n=3)$. Densitometry analysis is provided next to the Western blot. Data are presented as mean \pm SEM. ${ }^{*} P<0.05$ by 1 -way ANOVA with post hoc Fisher's least significant difference (LSD) test.

LsTtp-KO mice have improved systemic insulin sensitivity after HFD. To study the metabolic effects of hepatic TTP in vivo, we generated ls Ttp-KO mice ( $A l b-\mathrm{Cre}^{+} ; \mathrm{Ttp}^{f l / f l}$, Figure 2, A and B) and subjected them to normal chow diet or HFD. WT and 1s Ttp-KO mice fed normal chow had similar body weight, fasting glucose, and fasting and fed insulin levels (Supplemental Figure 2, A-D). Additionally, WT and 1s TtpKO mice fed normal chow displayed comparable insulin sensitivity - as evidenced by similar glucose and insulin tolerance, as well as glucose-induced insulin secretion (Figure 2, C-E).

To determine the effect of hepatic TTP on diabetes, we fed HFD to age-matched male WT and 1s Ttp-KO littermates. Despite similar body weight, fasting blood glucose, and serum insulin levels between WT and 1s Ttp-KO mice after 12 weeks of HFD (Supplemental Figure 2, A-D), 1s Ttp-KO mice had improved glucose tolerance and insulin sensitivity (Figure 2, C and D). This improvement was not due to differences in weight gain or changes in body composition, as 1s Ttp-KO mice had similar body weight as WT mice after HFD, and proton nuclear magnetic resonance performed at 15 weeks after HFD demonstrated similar body fat composition between WT and 1sTtp-KO mice (Figure 2, F and G, and Supplemental Figure 2E). Consistent with improved insulin sensitivity, 1s Ttp-KO mice fed HFD for 10 weeks demonstrated reduced insulin secretion in response to a glucose bolus (Figure 2E) compared 

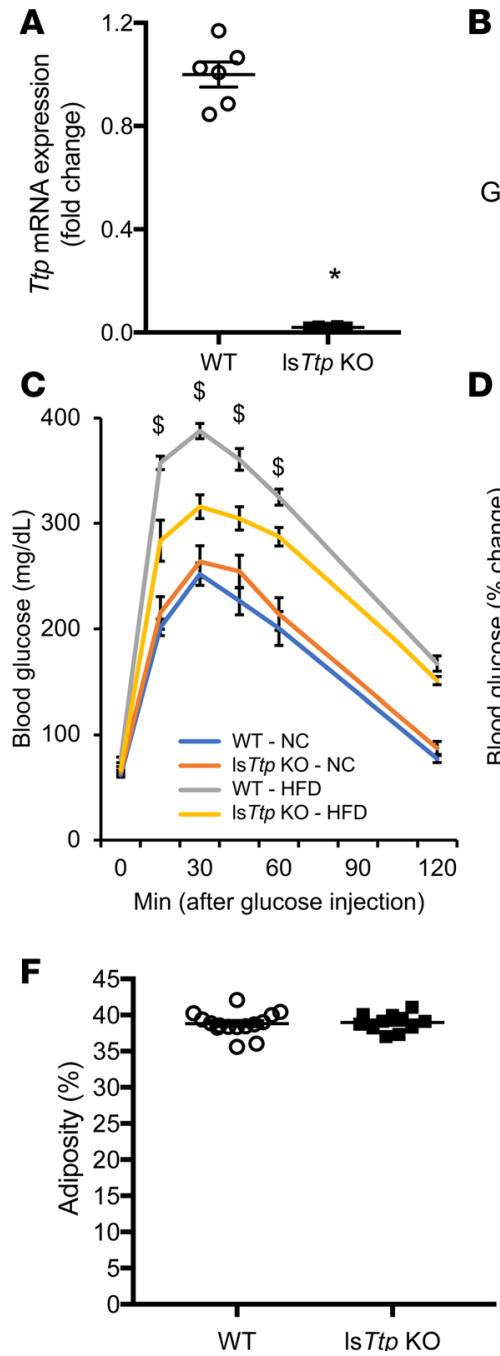

B

D
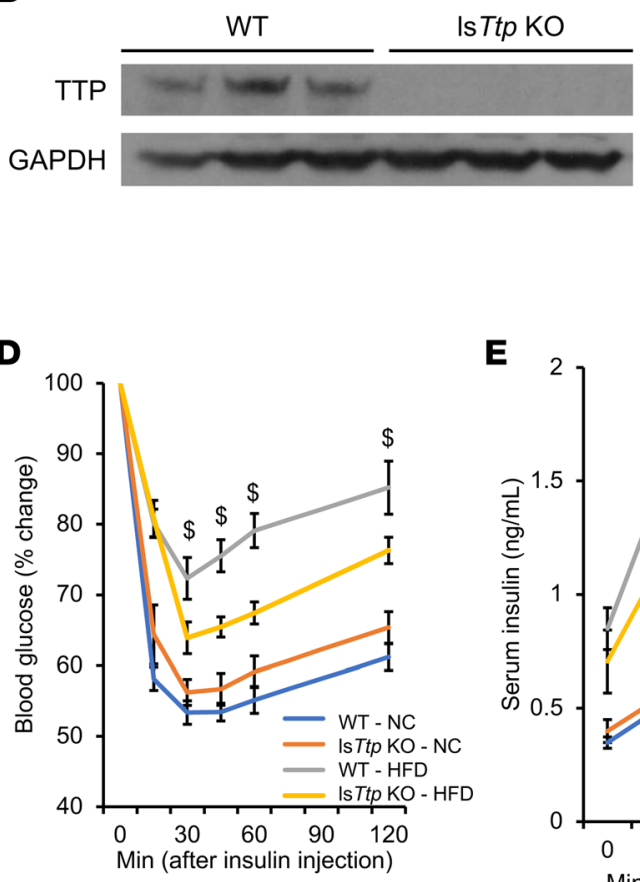

E
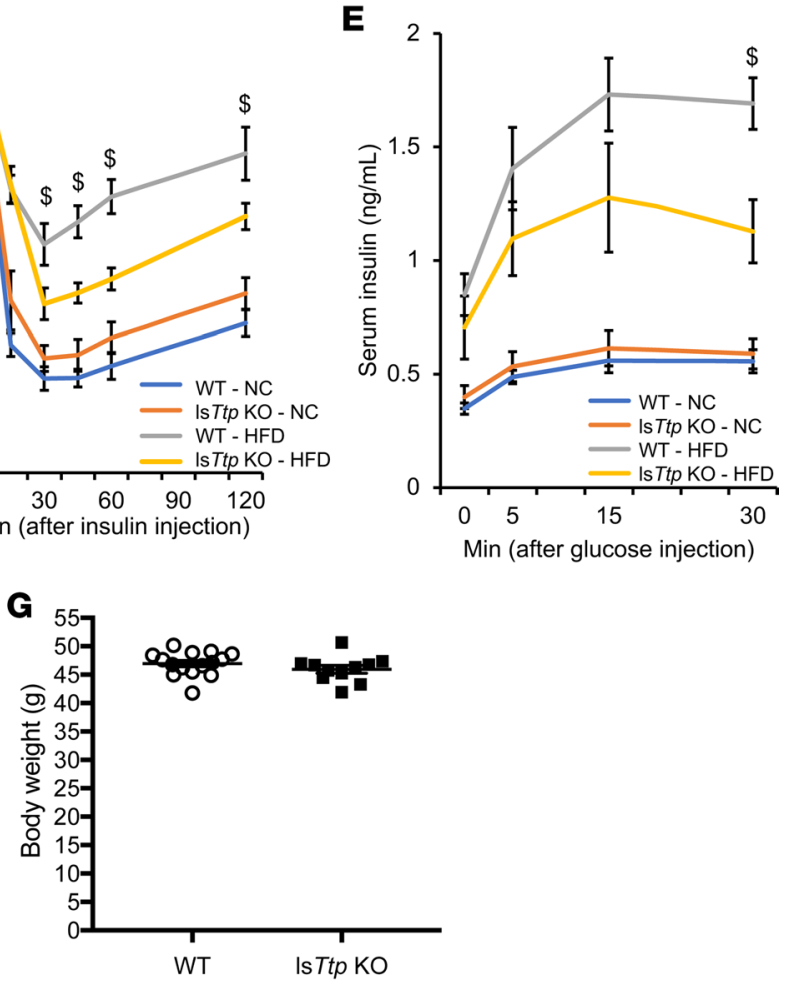

Figure 2. Deletion of Ttp in the liver results in improved glucose tolerance and insulin sensitivity after HFD. (A and B) Hepatic Ttp mRNA (A) and protein (B) levels in WT and IsTtp-KO mice. $n=4$ for $\mathbf{A}$ and 3 for B. ${ }^{*} P<0.05$ by 2 -tailed unpaired Student's $t$ test. (C) IsTtp-KO mice have improved glucose tolerance after 8 weeks of HFD compared with WT mice fed HFD. (D) IsTtp-KO mice have improved insulin sensitivity after 9 weeks of HFD compared with WT mice fed HFD. (E) IsTtp-KO mice have less glucose-stimulated insulin secretion after 10 weeks of HFD compared with WT mice fed HFD. ${ }^{\$} P<0.05$ compared with WT mice after HFD by 1-way ANOVA with post hoc Fisher's LSD test. NC, normal chow; HFD, high-fat diet; $n=7$ for WT and IsTtp-KO fed NC, 6 for WT, and 10 for IsTtp-KO fed HFD for C and D; $n=7$ for WT, 8 for IsTtp-KO fed NC, 6 for WT, and 9 for IsTtp-KO for E. (F) Adiposity as determined by fat mass percentage in WT and IsTtp-KO mice after 15 weeks of HFD. (G) Body weight of WT and IsTtp-KO mice after 15 weeks of HFD. $n=6-10$ for each group for $\mathbf{F}$ and $\mathbf{G}$. Data are presented as mean \pm SEM.

with control mice. Additionally, 1s Ttp-KO mice challenged with sodium octanoate injection exhibited increased ketone body levels after 2 hours compared with control mice, suggesting increased ketogenic potential in 1sTtp-KO mice after HFD (Supplemental Figure 2F).

We then examined whether the improved insulin sensitivity in 1sTtp-KO mice is due to differences in hepatic and circulating triglycerides (TG) or non-esterified FA (NEFA) levels. While fasting serum NEFA, serum TG, and hepatic TG were reduced in lean 1sTtp-KO mice compared with lean WT mice (Figure 3, A-C), no significant change in fasting hepatic TG levels, fasting serum TG, or fasting or fed serum NEFA was observed between WT and 1sTtp-KO mice after HFD (Figure 3, D-G). Therefore, the protective effect of hepatic Ttp deletion cannot be attributed to differences in hepatic lipid handling.

Because we observed no change in hepatic lipid handling, we hypothesized that the improved insulin sensitivity in 1sTtp-KO mice may be due to an extrahepatic effect, such as changes in peripheral tissue insulin sensitivity. Peripheral tissue insulin sensitivity was increased in 1sTtp-KO mice compared with WT mice after HFD, as assessed by insulin-induced phosphorylated AKT (pAKT) levels in the epididymal AT (eAT), skeletal muscle, and heart (Figure 4, A-D, and Supplemental Figure 3A). We also observed higher insulin-induced 
Lean
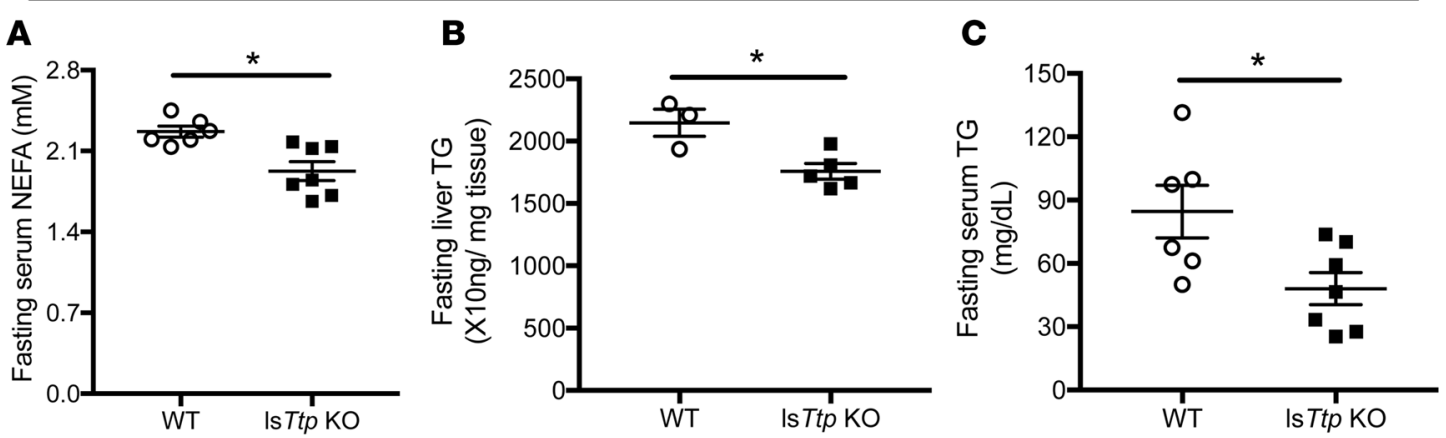

HFD
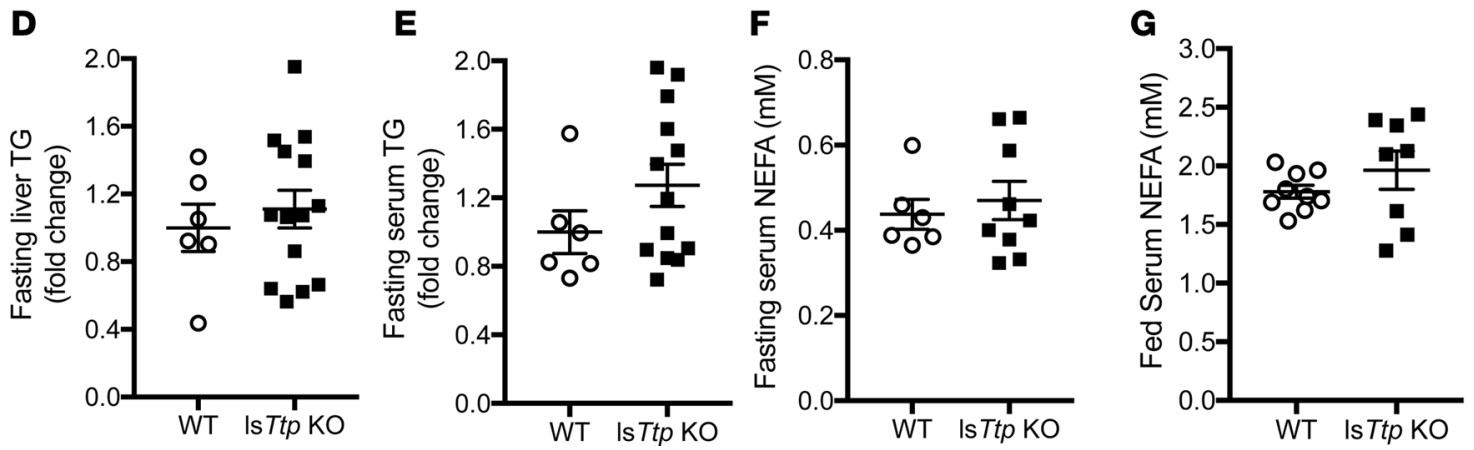

Figure 3. Liver-specific deletion of Ttp results in changes in lipid parameters at baseline but not after HFD. (A-C) Fasting serum non-esterified fatty acid (NEFA) (A), hepatic triglyceride (B), and serum triglyceride (C) in WT and IsTtp-KO mice fed normal chow. (D and E) Fasting liver (D) and serum (E) triglyceride in WT and IsTtp-KO mice after HFD. (F and $\mathbf{C})$ Fasting (F) and fed (C) serum NEFA in WT and IsTtp-KO mice after HFD. $n=4-9$ for each group. Data are presented as mean \pm SEM. ${ }^{*} P<0.05$ by 2-tailed unpaired Student's $t$ test.

AKT phosphorylation in the liver (Supplemental Figure 3B). In hyperinsulinemic-euglycemic clamp studies, 1sTtp-KO mice fed HFD demonstrated significantly lower basal and clamped insulin levels compared with control littermates (Figure 4E). Additionally, 1sTtp-KO mice demonstrated slightly lower blood glucose without a significant difference in glucose infusion rate (Supplemental Figure 4, A and B). Despite lower basal insulin levels, 1sTtp-KO mice achieved similar suppression of endogenous glucose production and had comparable glucose disposal rates (Supplemental Figure 4, C-F), indicating that lsTtp-KO mice require less insulin to achieve similar glucose clearance in the peripheral tissues. These data confirm that HFD-fed lsTtp-KO mice exhibit an increase in hepatic and peripheral insulin sensitivity compared with WT mice.

TTP binds to and posttranscriptionally represses Fgf21. The above data is most consistent with TTP regulating the expression of a liver-derived secretory factor that contributes to hepatic and peripheral insulin sensitivity. We therefore performed an in silico analysis of hepatic secretory proteins for the presence of TTP binding sites in their $3^{\prime}$-UTR. Of the 244 proteins that are predicted or confirmed to be secreted by the liver, 13 candidate genes were identified to have optimal nonamer TTP binding AREs (UUAUUUAUU) in the 3'-UTR of both human and mouse isoforms (Supplemental Table 2). Quantitative PCR (qPCR) analysis in 1sTtp-KO primary hepatocytes identified 6 transcripts to be significantly upregulated (Supplemental Figure $5 \mathrm{~A})$. We then measured the hepatic expression of these genes from 1sTtp-KO and control mice at baseline and after HFD. Among the 6 transcripts, only Fgf21 and follistatin-related protein 3 (Fstl3) were significantly upregulated in 1sTtp-KO mice both at baseline and after HFD (Figure 5, A and B, and Supplemental Figure 5, B and C). Since deletion of Fstl3 has been linked to increased insulin sensitivity (22), its upregulation in TTP-deleted mice would not explain the increased insulin sensitivity noted in these mice. On the other hand, pharmacological administration of FGF21 leads to improved insulin sensitivity $(23,24)$. Thus, we focused our studies on Fgf21. Consistent with higher hepatic Fgf21 mRNA levels and the fact that liver is the predominant site of Fgf21 production (25), serum levels of FGF21 after a 16-hour fast were significantly increased in 1sTtp-KO mice compared with control mice at baseline and after HFD (Figure 5C). 
A

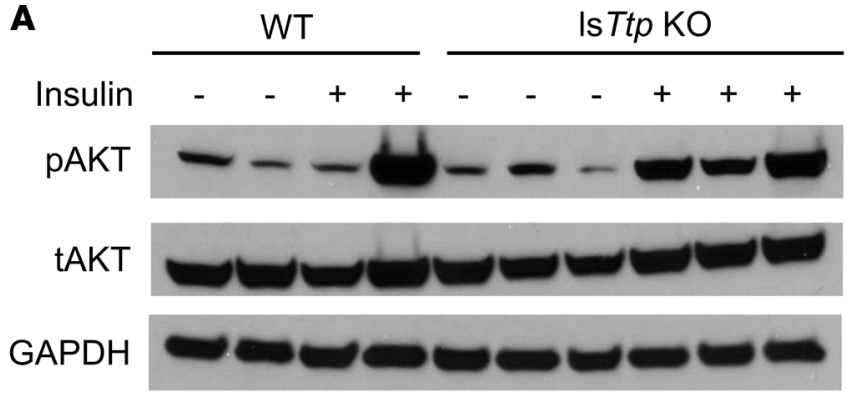

C
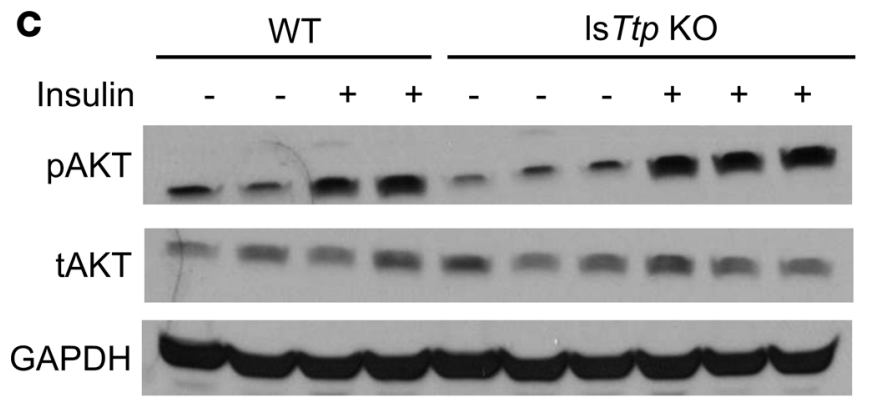

B

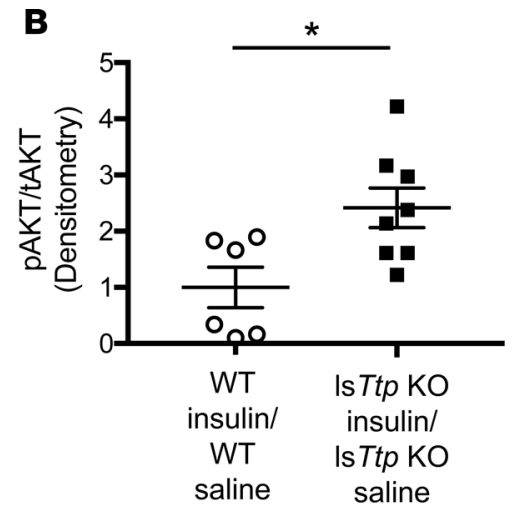

D

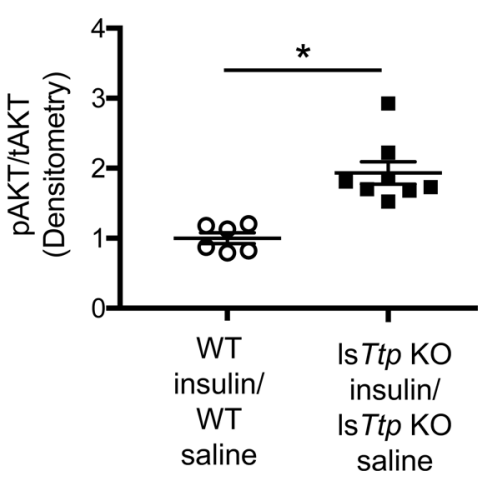

E

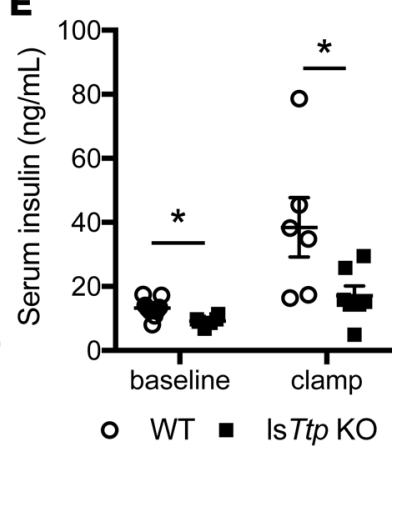

Figure 4. Liver-specific Ttp-KO mice have increased peripheral tissue insulin sensitivity after high-fat diet. (A-D) IsTtp-KO mice have improved insulin sensitivity in adipose tissue (A and B) and skeletal muscle (C and $\mathbf{D}$ ) after 12 weeks of HFD compared with control mice, as assessed by phosphorylated AKT 5473 (pAKT) to total AKT (tAKT) ratio after injection of insulin $(0.0075 \mathrm{U} / \mathrm{kg})$ or saline $(n=6-9)$. Representative Western blotting results are shown in $\mathbf{A}$ and $\mathbf{C}$, and densitometry analyses are provided in $\mathbf{B}$ and $\mathbf{D}$. (E) IsTtp-KO mice fed HFD have significantly lower insulin levels at baseline and during hyperinsulinemic-euglycemic clamp studies. Data are presented as mean \pm SEM. ${ }^{*} P<0.05$ by 2-tailed unpaired Student's $t$ test.

We then conducted additional studies to determine if FGF21 mRNA is a target of TTP. We identified several AREs in the FGF21 3'-UTR that are highly evolutionarily conserved across species (Figure 5D). Additionally, primary hepatocytes from 1sTtp-KO mice treated with the transcriptional inhibitor actinomycin D demonstrated slower degradation of Fgf 1 mRNA compared with those from primary hepatocytes from WT mice, consistent with posttranscriptional regulation of $F g f 21$ by TTP (Figure 5E). To assess the interaction between TTP protein and FGF21 mRNA, we performed an RNA co-IP using anti-human TTP or control IgG antibody and analyzing mRNA targets of TTP by qPCR. We observed enrichment in a known target of TTP (VEGFA) in the TTP antibody group compared with IgG, and we observed no enrichment for the negative control (hypoxanthine phosphoribosyltransferase 1; HPRT1) (Figure 5F). FGF21 mRNA levels were significantly enriched when incubated with TTP antibody compared with IgG control, indicative of an interaction between TTP protein and FGF21 mRNA (Figure 5F).

To determine whether TTP regulates FGF21 mRNA at the level of its 3'-UTR, we cloned the fulllength 3'-UTR of human FGF21 downstream of the firefly luciferase gene (FGF21 3'-UTR-luc). Consistent with TTP suppression of FGF21 mRNA at its 3'-UTR, transfection of the FGF21 3'-UTR-luc construct into WT MEFs led to a significant reduction in the luminescence signal compared with the same reporter construct in Ttp-KO MEFs (Figure 5G). We then assessed whether direct binding of TTP to ARE sequences is required for its function by overexpressing WT TTP or C124R TTP (mutation in the TZF, rendering TTP unable to bind to AREs) in HEK293 cells transfected with the FGF21 3'-UTRluc construct. While TTP overexpression resulted in a decrease in luminescence signal, this was rescued to baseline levels with overexpression of C124R TTP, confirming that TTP requires its TZF domain to bind to the FGF21 $3^{\prime}$-UTR and promote its degradation (Figure 5H). A similar effect was observed with mouse Fgf21 3'-UTR-luc construct transfected into WT and Ttp-KO MEFs and into Ttp-KO MEFs overexpressing WT or TZF mutant TTP (Supplemental Figure 5, D and E). Additionally, deletion of ARE 
A

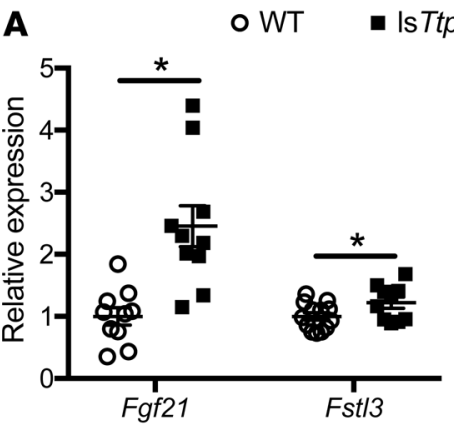

B

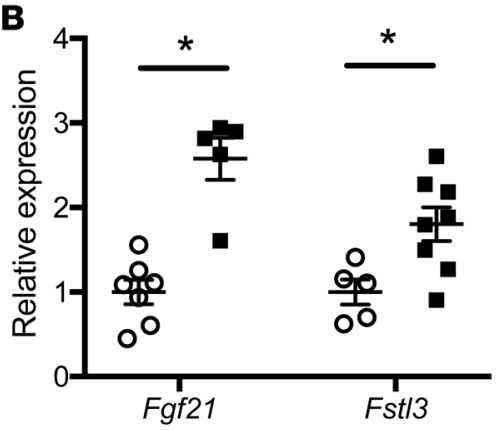

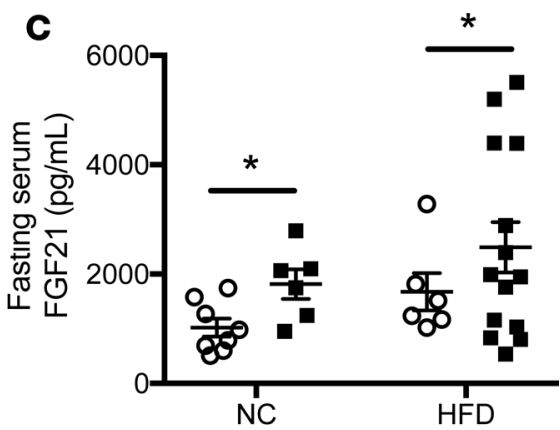

D

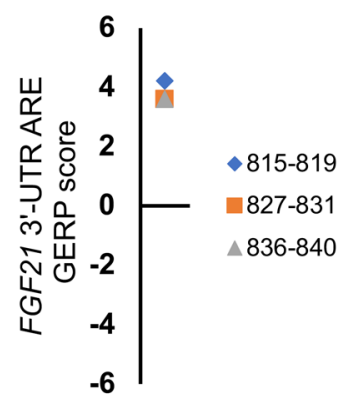

G

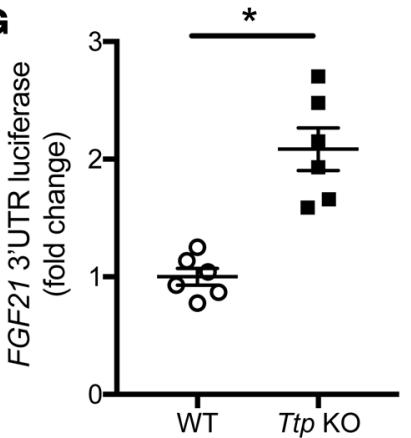

E

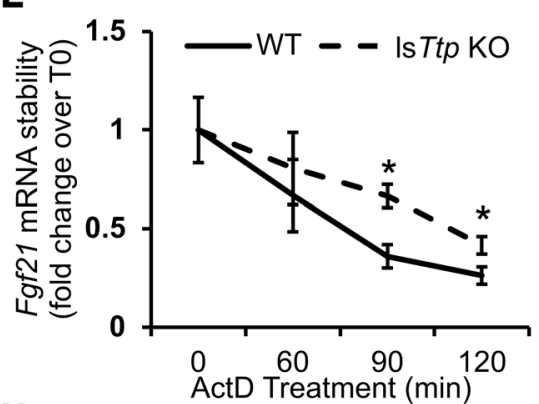

H

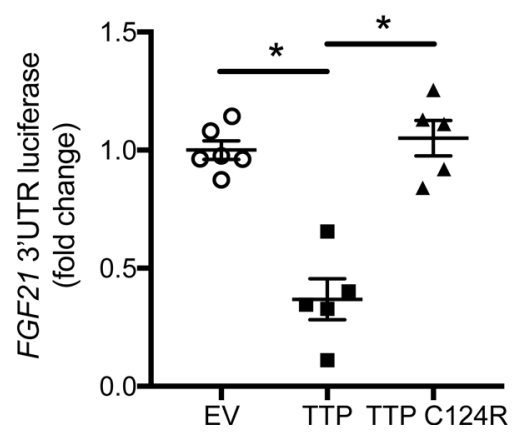

$\mathbf{F}$

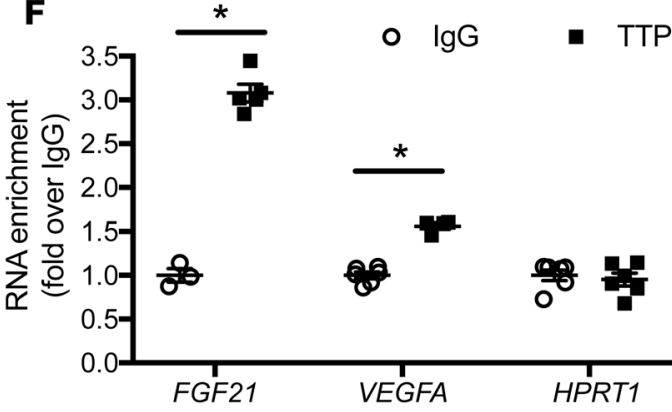

I

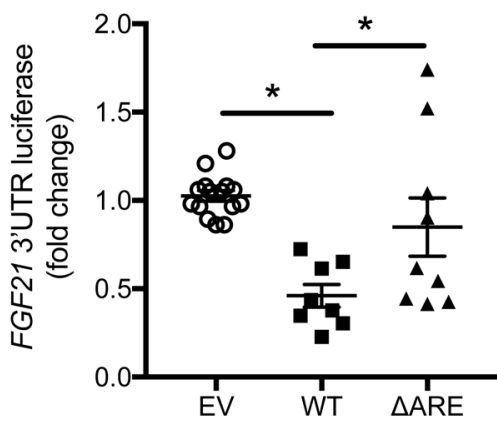

Figure 5. Fgf21 is a target of TTP. (A) mRNA expression of putative Ttp-targeted hepatic secretory proteins in liver from WT and IsTtp-KO mouse fed normal chow $(n=10-12)$. (B) mRNA levels of putative Ttp-targeted hepatic secretory proteins in liver from WT and IsTtp-KO mouse fed HFD ( $n=6-9)$. (C) Fasted IsTtp-KO mice have increased circulating FGF21 levels after 12 weeks of NC or HFD compared with WT mice $(n=6-14)$. (D) Genomic Evolutionary Rate Profiling (GERP) of AU-rich elements (AREs) in the FGF21 transcript demonstrates several evolutionarily conserved AREs. The numbers in the legend represent the nucleotide location of the ARE site with respect to the start of the coding region. (E) Fgf21 mRNA is stabilized in primary hepatocytes from IsTtp-KO compared with WT mice $(n=4)$. (F) TTP protein physically interacts with FGF21 mRNA as assessed by RNA co-IP $(n=3-6)$. (G) Human FGF21 3'-UTR-luciferase reporter construct signal is higher in Ttp-KO MEFs compared with WT MEFs $(n=6)$. (H) Overexpression of WT TTP, but not C124R mutant TTP, reduces luminescence signal of the human FGF21 3'-UTR-luciferase reporter construct $(n=5-6)$. (I) Deletion of ARE in human FGF21 3'-UTR-luciferase construct ( $\triangle \mathrm{ARE}$ ) abolishes the regulation by TTP overexpression $(n=8-15)$. Data presented as the relative luciferase activity in the presence of TTP overexpression for each construct. NC, normal chow; HFD, high-fat diet; EV, empty pMIR-REPORT vector; WT, WT human FCF21 3'-UTR-luciferase construct. Data are presented as mean \pm SEM. ${ }^{*} P<0.05$ by 1 -way ANOVA with post hoc Fisher's LSD test.

sequences in human and mouse FGF21 3'-UTR-luc constructs abolished their response to TTP overexpression (Figures 5I and Supplemental Figure 5F). These results indicate that TTP posttranscriptionally regulates FGF21 expression directly by binding to the ARE in its $3^{\prime}$-UTR.

Hepatic deletion of Ttp affects gene transcription in muscle and AT, and it leads to brown fat activation. To assess the peripheral effects of increased FGF21 in 1sTtp-KO mice, we measured Adipoq (which encodes the adipokine adiponectin) gene expression levels in various AT depots. LsTtp-KO mice had significantly higher Adipoq mRNA levels in eAT and inguinal AT (iAT) compared with WT control mice (Figure 6A). Consistent with reports of improved hepatic and muscle insulin sensitivity with increased adiponectin (26), 1sTtp-KO mice fed HFD demonstrated increased AKT phosphorylation upon insulin stimulation compared with control mice (Figure 4, C and D, and Supplemental Figure 3B). Increased circulatory FGF21 has also been shown to contribute to peripheral insulin sensitivity through increased glucose uptake and oxidation in muscle and brown $\mathrm{AT}$ (bAT) $(27,28)$. Consistent with this mechanism, glucose 
A $\quad \circ \quad W T=$ IsTtp KO

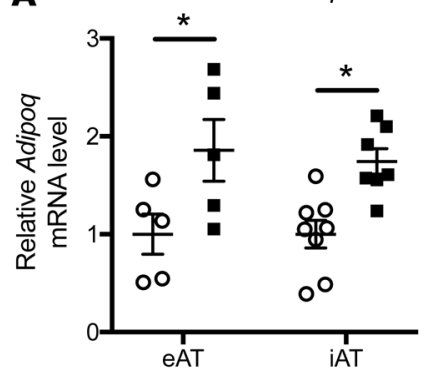

D
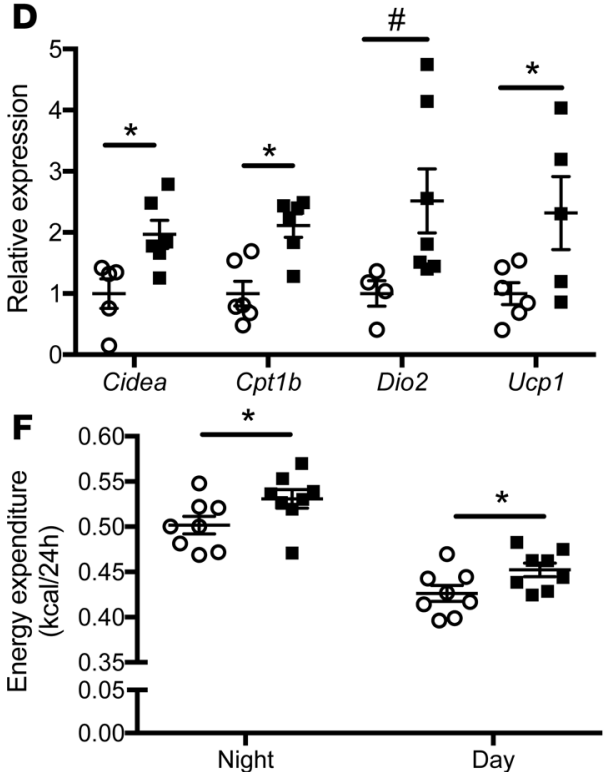
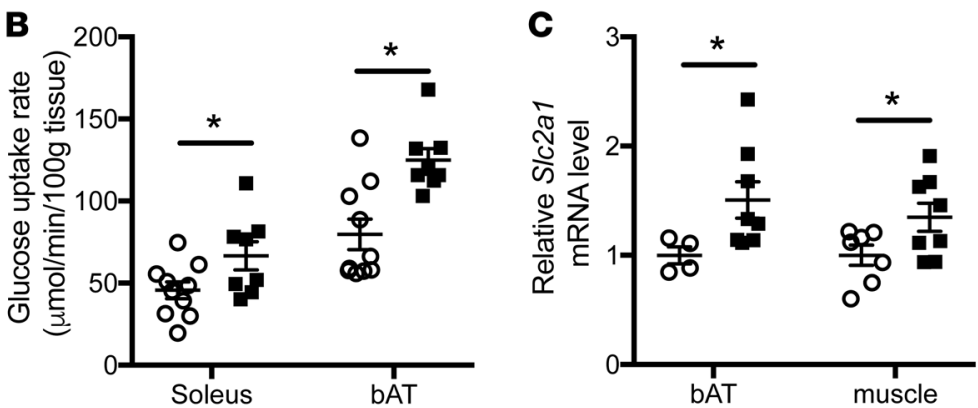

$\mathbf{E}$
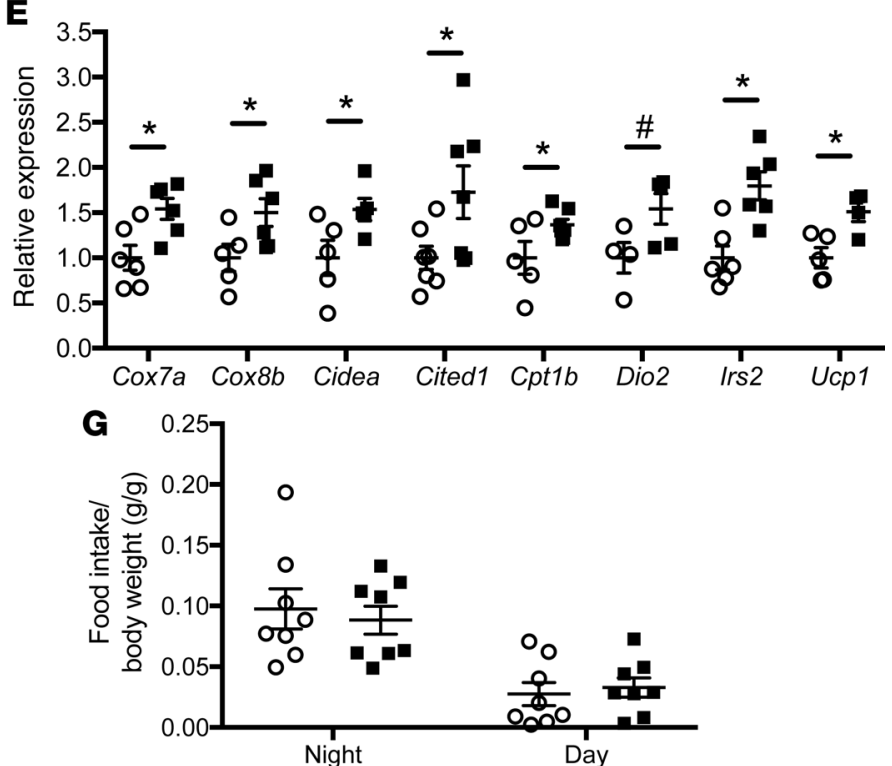

Figure 6. Hepatic deletion of Ttp activates brown and beige adipose tissue. (A) Adipoq mRNA levels in epididymal adipose tissue (eAT) and inguinal AT (iAT) from WT and IsTtp-KO mice fed HFD $(n=5-8)$. (B) Soleus muscle and brown adipose tissue (bAT) glucose uptake at the end of hyperinsulinemic-euglycemic clamp studies in WT and IsTtp-KO mice fed HFD $(n=7-10)$. (C) SIc2a1 expression in bAT and skeletal muscle from WT and IsTtp-KO mice after HFD $(n=4-8)$. (D) Loss of hepatic Ttp results in a gene expression pattern in iAT consistent with beige adipose tissue browning $(n=4-7)$. (E) Gene expression in bAT in IsTtp-KO mice is consistent with increased brown adipose tissue activation $(n=4-7)$. (F) IsTtp-KO mice have increased energy expenditure after HFD $(n=6-8)$. (C) Food intake of WT and IsTtp-KO mice during indirect calorimetry $(n=6-8)$. Data are presented as mean \pm SEM. ${ }^{*} P<0.05,{ }^{\#} P<0.1$ by 2 -tailed unpaired Student's $t$ test.

uptake in bAT and soleus muscle was significantly increased in HFD-fed 1sTtp-KO mice compared with WT littermates (Figure 6B) and was accompanied by increased adipose and muscle Slc2al (which encodes GLUT1 transporter) expression (Figure 6C).

At the molecular level, iAT (a beige AT depot) from 1sTtp-KO mice after HFD demonstrated significantly higher mRNA levels of Cidea, Ucp1, and Cpt1b and a trend toward increasing Dio2 mRNA levels compared with control mice (Figure 6D). In bAT, we also observed significantly higher levels of Cox7a, Cox8b, Cpt1b, Cidea, Cited1, Ucp1, and Irs2 and a trend toward increasing Dio2 mRNA in 1sTtp-KO mice compared with control mice (Figure 6E). These observations are consistent with increased thermogenic potential in brown fat and increased browning of beige AT in 1sTtp-KO mice compared with control mice. Finally, indirect calorimetry revealed a significant increase in energy expenditure in 1sTtp-KO mice after HFD (Figure 6F), consistent with increased bAT activity. No difference in food intake was observed (Figure 6G). Taken together, our data indicate that the baseline increase in circulating FGF21 in 1sTtp-KO mice (Figure 5C) promotes brown fat activation, peripheral glucose uptake, and adiponectin production after HFD to protect against the development of diabetes.

Hepatic TTP regulates systemic metabolism through modulation of Fgf21. To confirm that hepatic FGF21 mediates the improved glucose tolerance and insulin sensitivity observed in 1sTtp-KO mice after HFD, we generated hepatotropic adeno-associated virus serotype 8 (AAV8) overexpressing shRNA against Fgf21 $(\mathrm{sh} F g / 21)$ or luciferase $(\mathrm{sh} L u c)$. Injection of AAV8 $\mathrm{sh} F g f 21$ into WT mice effectively reduced circulating 

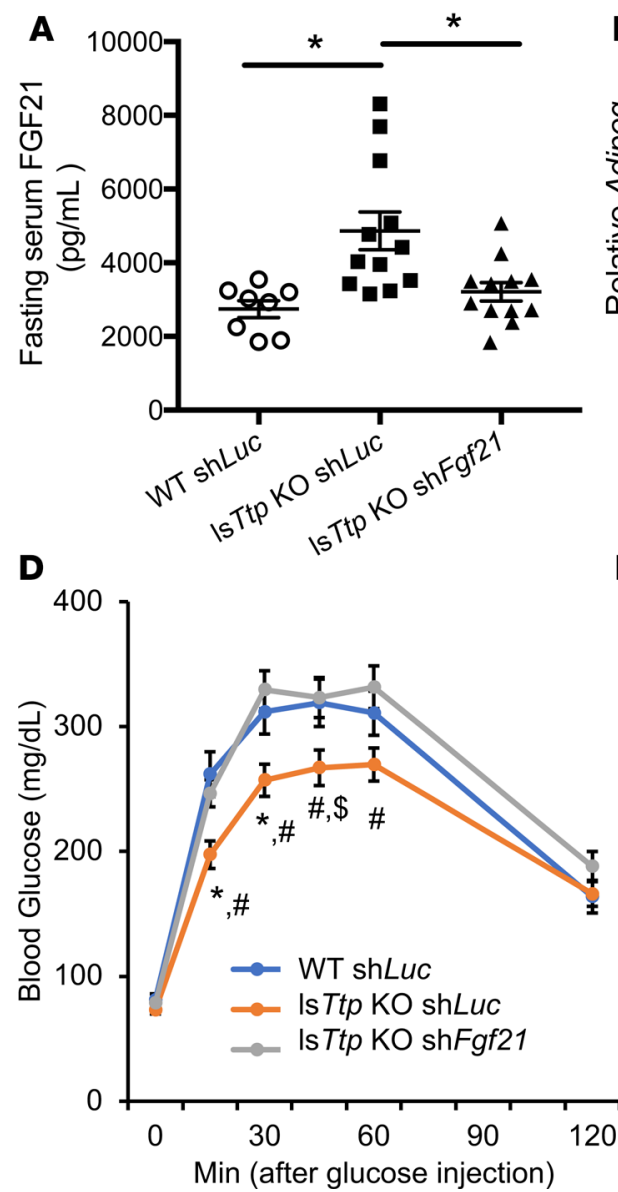

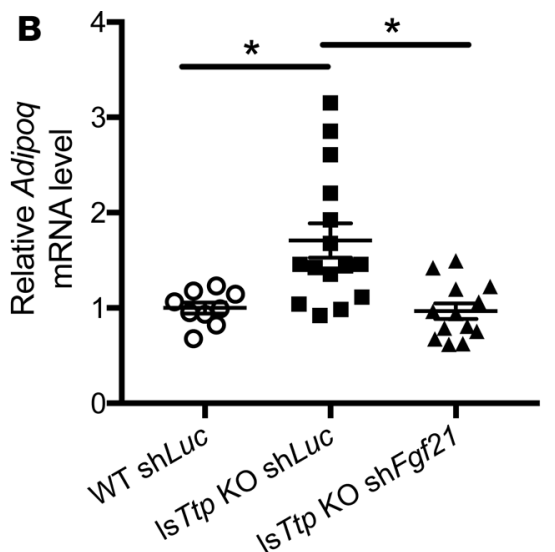

$\mathbf{E}$

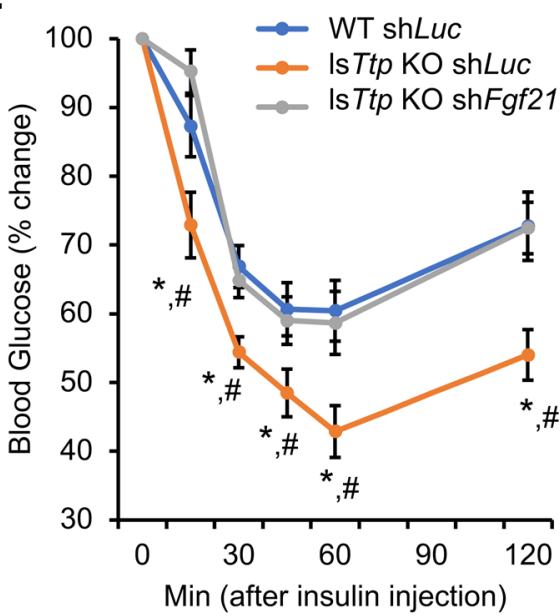

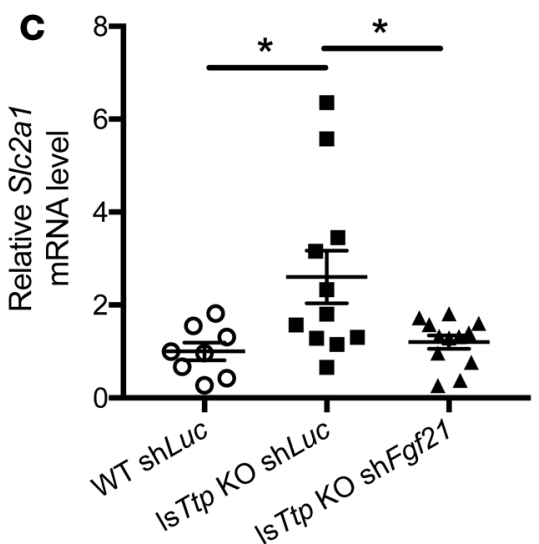

$\mathbf{F}$

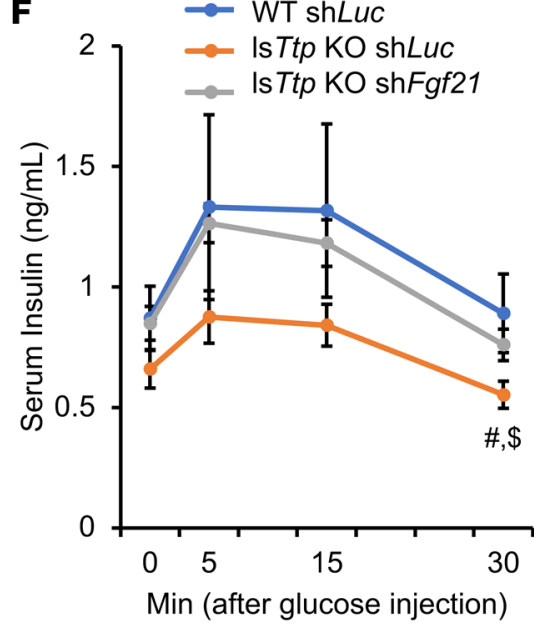

Figure 7. Increased liver-derived circulating FGF21 is responsible for improved systemic diabetic parameters after high-fat diet in the setting of hepatic Ttp deletion. (A) Fasting levels of circulating FGF21 are effectively reduced in IsTtp-KO mice injected with a hepatotropic Fgf21 shRNA AAV8 virus (shFgf21) at 3 weeks of age and then fed HFD for 12 weeks. (B and C) Downregulation of Fgf21 in IsTtp-KO mice leads to normalization of Adipoq mRNA levels in adipose tissue (B) and Slc2a1 mRNA levels in skeletal muscle (C) after HFD; $n=8$ for WT shLuc and 12 for IsTtp-KO shLuc and shFgf21. ${ }^{*} P<0.05$ by 1 -way ANOVA with post hoc Fisher's LSD test. (D-F) IsTtp-KO mice injected with Fgf21 shRNA AAV8 have reversal of glucose tolerance (D), insulin sensitivity (E), and glucose-stimulated insulin secretion (F) after HFD compared with IsTtp-KO mice injected with shLuc ( $n=7$ for WT shLuc, 9 for IsTtp-KO shLuc, and 16 for IsTtp-KO shFgf21 for D-F) ${ }^{*} P<0.05$ between IsTtp-KO shFgf21 and IsTtp-KO shLuc, ${ }^{*} P<0.05$ between IsTtp-KO shLuc and WT shLuc, ${ }^{\$} P<0.1$ between IsTtp-KO shLuc and WT shLuc. Data are presented as mean \pm SEM, using 1-way ANOVA with post hoc Fisher's LSD test.

FGF21 levels (Supplemental Figure 6A). We injected the virus into WT and 1sTtp-KO mice at 3 weeks of age, followed by HFD treatment for 12 weeks. At the end of HFD treatment, we observed normalization of hepatic Fgf21 expression and circulating FGF21 levels in 1s Ttp-KO mice injected with AAV8 shFgf21 (Figure 7A and Supplemental Figure 6B). No change in AT Fgf21 expression was observed in 1sTtp-KO mice injected with AAV8 shFgf21 (Supplemental Figure 6C), confirming that changes in circulating FGF21 levels were due to hepatic Fgf21 expression. Additionally, downregulation of Fgf21 in 1s Ttp-KO mice also normalized AT Adipoq mRNA levels (Figure 7B), as well as Slc2a1 expression in skeletal muscle (Figure 7C). Knockdown of Fgf21 in 1sTtp-KO mice fed HFD led to reversal of glucose tolerance, glucose-stimulated insulin secretion (GSIS), ketogenesis, and systemic insulin sensitivity (Figure 7, D-F, and Supplemental Figure 6), demonstrating that increased circulating liver-derived FGF21 is responsible for the improved systemic diabetic parameters in 1s Ttp-KO mice fed HFD.

\section{Discussion}

The liver plays a critical role in regulating whole body energy homeostasis via its ability to utilize a variety of substrates in response to nutritional challenges and to secrete factors that modulate systemic metabolism. In this study, we show that TTP, an RNA-binding protein previously studied in the fields of inflammation, cancer, and cellular iron homeostasis, plays a potentially novel role in hepatic metabolism and systemic insulin sensitivity (12-14). We show that hepatic TTP is induced by insulin in vitro and in vivo 
and that TTP levels are reduced in the livers of diabetic mice and humans, likely due to hepatic insulin resistance. LsTtp-KO mice have improved glucose tolerance and peripheral insulin sensitivity after HFD compared with littermate controls. Analysis of liver-secreted factors demonstrated that TTP binds to and promotes the degradation of FGF21 mRNA. Finally, we show that knockdown of Fgf21 in 1sTtp-KO mice fed HFD leads to reversal of systemic glucose tolerance and insulin sensitivity, confirming that hepatic TTP regulates systemic metabolism through modulation of liver-derived FGF21.

Consistent with previous reports $(15,18)$, we show that hepatic TTP levels are reduced in diet-induced and genetic murine models of obesity and in human diabetic livers. We also show that TTP protein is robustly induced by insulin injection in WT mice, but not in diabetic mice, demonstrating that intact insulin sensitivity is necessary for the induction of TTP by insulin. This suggests that the reduced baseline levels of hepatic TTP expression in diabetic mice and humans may be due to increased basal hepatic insulin resistance.

It is intriguing that late loss of hepatic TTP (as observed in WT diabetic mice) coincides with glucose intolerance and insulin resistance, yet early loss of hepatic TTP (resulting from genetic deletion in 1s Ttp$\mathrm{KO}$ mice) leads to improved diabetic parameters after HFD compared with control mice. While FGF21 is elevated in both conditions, it has been proposed that upregulation of FGF21 after the onset of diabetes is insufficient to improve insulin sensitivity $(29,30)$. These observations suggest that lower basal hepatic TTP expression in the healthy state promotes systemic insulin sensitivity due to diminished repressive effects on FGF21. This would, in turn, allow the body to withstand the diabetic insults of peripheral tissue insulin resistance and delay the onset of disease. Conversely, repression of TTP secondary to established diabetes is likely due to insulin resistance and diminished AKT signaling regulating TTP at the promoter level. It is interesting to speculate that high baseline hepatic TTP expression in humans, due to natural variation or TTP polymorphisms, may lead to reduced basal levels of systemic insulin sensitivity, rendering those individuals ill-equipped to withstand the metabolic changes associated with obesity and resulting in a more rapid progression to diabetes.

FGF21 is a complex hepatic hormone that is highly regulated by various metabolic challenges, including fasting, high-fat, low-carbohydrate, low-protein, and low-essential amino acid diets (1, 31-33). FGF21 plays an important role in coordinating the peripheral response to insulin, as genetic deletion of FGF21 leads to peripheral insulin insensitivity despite mice being normoglycemic (34). Circulating FGF21 is important for the production of adiponectin from various AT depots (35), which in turn modulates insulin sensitivity in the liver and skeletal muscle. Additionally, FGF21 signaling leads to increased glucose uptake in skeletal muscle, as well as in beige AT and bAT $(28,36)$. It is also required for the adaptation to cold temperature by inducing beige AT browning and increasing thermogenic potential in bAT $(36,37)$. In fact, serum FGF21 levels have been linked to bAT activity in lean healthy subjects (38). In addition to its effect on AT, liver-specific Fgf21 transgenic mice have lower serum insulin, cholesterol, TG, and hepatic TG levels (39). Administration of FGF21 in $o b / o b$ mice also leads to a reduction in serum TG levels $(23,40)$. Similar to these reports, we also observe increased adiponectin expression, peripheral insulin sensitivity, bAT activation, and peripheral tissue glucose uptake in 1s Ttp-KO mice compared with control mice.

While our results generally agree with the literature, there are a few distinctions. Although we observed reduced serum NEFA and TG in lean 1s Ttp-KO mice, no difference in serum NEFA, TG, or liver TG was seen in 1s Ttp-KO mice after HFD compared with control mice. This is in contrast with previous findings of FGF21 administration to $o b / o b$ mice (41). Additionally, mice receiving pharmacological doses of FGF21 also demonstrated weight loss and changes in fat mass $(4,41,42)$; however, 1s Ttp-KO mice at baseline or after HFD did not have a significant difference in body weight compared with littermate controls (Figure $2 \mathrm{G}$ and Supplemental Figure 2A). Fat mass was also not significantly different between 1sTtp-KO mice and control mice after HFD (Figure 2F and Supplemental Figure 2E). These distinctions between our findings and previous studies may be attributed to the fold induction of FGF21; 1s Ttp-KO mice typically achieve about a 2- to 4-fold increase in FGF21 over control mice, while previous reports using pharmacological doses of FGF21 can lead to more than a 20-fold increase in FGF21 $(41,43)$. While supraphysiologic doses of FGF21 may achieve weight loss and increase insulin sensitivity, our findings highlight that a physiological increase of FGF21 prior to the onset of diabetes can also be protective.

While basal hepatic expression of FGF21 is low, its expression is significantly induced up to 100-fold by changes in nutrition status (32). In our study, 1s Ttp-KO mice fed normal chow showed a 2-fold increase in serum FGF21 levels compared with WT control mice. This modest increase in FGF21, consistent with 
prior reports of TTP acting to fine-tune cellular processes (14), and the fact that mice fed normal chow are already at a near maximally insulin-sensitive state likely explains the lack of improvement in insulin sensitivity in 1s Ttp-KO mice at baseline. However, we show that the difference in serum FGF21 levels between 1s Ttp-KO and WT mice is magnified after HFD and is sufficient to account for the improvement in insulin sensitivity of HFD-fed 1sTtp-KO mice through its effects on insulin action and peripheral glucose utilization. Thus, the TTP-mediated regulation of $F g f 21$ mRNA may play a critical role in insulin sensitivity under diseased conditions, such as diabetes, but not at baseline.

Although FGF21 mimetics have shown promise in human clinical trials in diabetic obese patients, their efficacy has been limited by the short half-life of FGF21 and the fact that obesity is ultimately an FGF21-resistant state $(29,30)$. While high levels of FGF21 in advanced diabetic patients serves as a marker of insulin resistance due to a reflexive but futile mechanism to improve insulin sensitivity, we show that high levels of FGF21 in our system are directly linked to hepatic TTP and serve as a driver of insulin sensitivity, as confirmed by reversal of our phenotype with knockdown of hepatic Fgf21. Thus, reducing hepatic TTP levels may be a novel therapeutic approach in metabolic syndrome and prediabetes, conditions with reduced yet intact insulin sensitivity, in order to directly increase FGF21 levels to prevent the onset of diabetes.

We identified FGF21 as a target of TTP by screening the murine and human transcripts of known or predicted liver-secreted proteins for the optimal TTP binding sequence UUAUUUAUU $(44,45)$. However, variations in the optimal nonamer binding sequence can be tolerated, and it is possible that this approach may have excluded some "true" TTP targets. Identification of novel target transcripts will better illustrate how TTP may lead to a sequential metabolic reprogramming at the hepatic and systemic levels.

In summary, our results identify hepatic TTP as a potentially novel player in metabolism that regulates the mRNA stability of Fgf21, which has functional consequences for hepatic and systemic insulin sensitivity. Furthermore, we show that hepatic TTP levels are induced by insulin and reduced in the livers of diabetic mice and humans. Finally, we show that 1s Ttp-KO mice have improved diabetic parameters after HFD compared with littermate controls and that hepatic TTP regulates systemic metabolism through modulation of FGF21. These findings may open up novel avenues in the therapeutic treatment of metabolic syndrome and prediabetes, particularly the posttranscriptional modulation of metabolic gene expression by RNA-binding proteins.

\section{Methods}

Mouse studies. WT C57BL/6 mice were purchased from Taconic. $d b / d b$ and $d b /+$ mice were purchased form the Jackson Laboratory. C57BL/6 Tt $t^{f / f l}$ mice (a gift from Perry Blackshear, National Institute of Environmental Health Sciences [NIEHS]) have flox sites flanking exon 2 of TTP. LsTtp-KO mice were generated by breeding $T t p^{f / f l}$ mice with albumin-Cre transgenic mice (a gift from Joseph Bass, Northwestern University). Ttp liver-specific deletion due to Cre-recombinase expression in hepatocytes was confirmed at the mRNA and protein levels. Only male mice were used in the studies because of the documented protection against HFD-induced insulin resistance in female mice (46).

AAV constructs containing shRNA against luciferase or Fgf21 were gifts from Roger Davis (University of Massachusetts, Amherst, Massachusetts, USA). The constructs were packaged into AAV serotype 8 viral particles by ViGene. Mice at 3 weeks of age were randomized to receive i.p. injection with $6 \times 10^{11}$ genome copies of AAV8 shLuc (control) or AAV8 shFgf21 in $200 \mu 1$ of normal saline.

For HFD studies, mice were fed with rodent chow consisting of $60 \%$ calories by fat, $20 \%$ calories by protein, and 20\% calories by carbohydrate (Research Diets, D14292) for indicated duration starting at 3 weeks of age. The researchers were not blinded to the genotype or the treatment of the mice.

Isolation and culturing of primary hepatocytes. After anesthetization with Avertin (created from freshly prepared tribromoethanol; Millipore Sigma), the portal vein of each mouse (males, $8-12$ weeks old) was cannulated with a $24 \mathrm{G} \times 3$ /4-inch i.v. catheter (Terumo), and the liver was perfused with $\mathrm{Ca}^{2+}-$ and $\mathrm{Mg}^{2+}$ free HBSS (Thermo Fisher Scientific) for 5 minutes, followed by perfusion DMEM containing 0.05\% type IV collagenase (MilliporeSigma) for 5 minutes. The perfusate was drained by an incision of the femoral artery. After perfusion, the liver was removed and placed in ice-cold DMEM (with $4.5 \mathrm{~g} / 1$ glucose and L-glutamine with no sodium pyruvate, Corning) and cells were gently released. The cell suspension was filtered through a 100- $\mu \mathrm{m}$ nylon strainer (BD Falcon, BD Biosciences) and centrifuged at $50 \mathrm{~g}$ for 1 minute. The cell pellets were washed 3 times with ice-cold DMEM (Corning) and then resuspended in DMEM containing $5 \%$ FBS (Atlanta Biologicals). Cell viability ( $~ 80 \%)$ was determined by trypan blue exclusion. 
Cells were plated on type I collagen-coated dishes at a density of $5 \times 10^{4} \mathrm{cells} / \mathrm{cm}^{2}$ and incubated at $37^{\circ} \mathrm{C}$ under $5 \% \mathrm{CO}_{2}$. After 1.5 hours of attachment, the media was aspirated, cells were washed with PBS and incubated in complete media (DMEM containing 10\% FBS and 1\% penicillin/streptomycin/amphotericin B; Corning). For insulin treatments, cells were incubated with complete media supplemented with recombinant human insulin expressed in yeast (MilliporeSigma) for the indicated times. For protein synthesis inhibition, cells were pretreated with cyclohexamide (MilliporeSigma, $0.1 \mathrm{mM}$ ) for 2 hours. All experiments involving cultured hepatocytes were completed within 48 hours of culturing.

Human diabetic liver samples. Diabetic and nondiabetic human liver samples were obtained via needle biopsy from obese patients during bariatric surgery at Northwestern University. Patient characteristics were provided in Supplemental Table 1. All patients were fasted prior to surgery.

Transfections. For overexpression experiments, human TTP and C124R mutant TTP (gifts from Perry Blackshear, NIEHS) constructs were transfected into MEF or HEK293 cells using Lipofectamine 2000 reagent (Invitrogen) in OptiMEM (Invitrogen) for 4 hours, followed by incubation in complete, antibiotic-free medium for another 20-44 hours, after which treatments were initiated.

In silico analysis of potential TTP target transcripts. The Human Protein Atlas (http://www.proteinatlas. $\mathrm{org} /$ ) was used to identify 244 predicted or confirmed secreted hepatic genes (47), which were screened for high-affinity nonamer AREs (UUAUUUAUU) in human and mouse transcripts using the AREsite program (http://rna.tbi.univie.ac.at/AREsite2/welcome) (48) or Ensembl Genome browser (http://useast. ensembl.org/index.html) (Supplemental Table 2). Real-time PCR was used to validate the expression levels of the 13 candidate genes in WT and 1sTtp-KO primary hepatocytes.

In vivo metabolic studies. For all in vivo metabolic studies, age-matched male WT and 1sTtp-KO littermates were used. For the glucose-tolerance test (GTT) and GSIS, mice were fasted for 16 hours and injected via an i.p. approach with a 20\% dextrose (MilliporeSigma) solution in PBS at $2 \mathrm{~g} / \mathrm{kg}$ body weight. For the insulin-tolerance test (ITT), mice were fasted for 4 hours and injected via an i.p. approach with a $0.1 \mathrm{U}$ Humulin/ml PBS solution at $0.75 \mathrm{U} / \mathrm{kg}$ body weight (Lily). For the ketogenesis challenge test, mice were fasted for 24 hours and injected via an i.p. approach with a $250 \mathrm{mM}$ sodium octanoate (MilliporeSigma) in deionized water and $\mathrm{pH}$ adjusted to 7.4 at $10 \mu \mathrm{g}$ body weight.

Hyperinsulinemic-euglycemic clamping and in vivo glucose uptake. Hyperinsulinemic-euglycemic clamping was performed at Mouse Metabolic and Phenotyping Core at Vanderbilt University. Catheters were implanted into a carotid artery and a jugular vein of mice for sampling and infusions, respectively, 5 days before the study as described by Berglund et al. (49). Insulin clamps were performed on mice fasted for 5 hours using a modification of the method described by Ayala et al. $(50)$. $\left[3-{ }^{3} \mathrm{H}\right]$-glucose was primed $(1.5 \mu \mathrm{Ci})$ and continuously infused for a 90 -minute equilibration and basal sampling periods $(0.075 \mu \mathrm{Ci} / \mathrm{min}) .\left[3-{ }^{3} \mathrm{H}\right]$-glucose was mixed with the nonradioactive glucose infusate (infusate specific activity of $0.5 \mu \mathrm{Ci} / \mathrm{mg}$ ) during the 2-hour clamp period. Arterial glucose was clamped using a variable rate of glucose (plus trace $\left[3-{ }^{3} \mathrm{H}\right]$-glucose) infusion, which was adjusted based on the measurement of blood glucose at 10-minute intervals. By mixing radioactive glucose with the nonradioactive glucose infused during a clamp, deviations in arterial glucose-specific activity are minimized and steady state conditions are achieved. The calculation of glucose kinetics is, therefore, more robust (51). Baseline blood or plasma variables were calculated as the mean of values obtained in blood samples collected at -15 and -5 minutes. At time zero, insulin infusion ( $4 \mathrm{mU} / \mathrm{kg}$ body weight $/ \mathrm{min}$ ) was started and continued for 120 minutes. Mice received heparinized saline-washed erythrocytes from donors at 5 $\mu \mathrm{l} / \mathrm{min}$ to prevent a fall in hematocrit. Blood was taken from 80-120 minutes for the determination of $\left[3-{ }^{3} \mathrm{H}\right]$-glucose. Clamp insulin was determined at $t=100$ and 120 minutes. At 120 minutes, $13 \mu \mathrm{Ci}$ of $2\left[{ }^{14} \mathrm{C}\right]$ deoxyglucose $\left(\left[{ }^{14} \mathrm{C}\right] 2 \mathrm{DG}\right)$ was administered as an i.v. bolus. Blood was taken from 2-25 minutes for determination of $\left[{ }^{14} \mathrm{C}\right] 2 \mathrm{DG}$. After the last sample, mice were anesthetized and tissues were freezeclamped for further analysis. Plasma insulin was determined by radioimmunoassay (RIA). Radioactivity of $\left[3-{ }^{3} \mathrm{H}\right]$-glucose and $\left[{ }^{14} \mathrm{C}\right] 2 \mathrm{DG}$ in plasma samples, and $\left[{ }^{14} \mathrm{C}\right] 2 \mathrm{DG}-6$-phosphate in tissue samples, were determined by liquid scintillation counting. Glucose appearance $(\mathrm{Ra})$ and disappearance $(\mathrm{Rd})$ rates were determined using steady state equations (52). Endogenous Ra (endoRa) was determined by subtracting the glucose infusion rate (GIR) from total Ra. The glucose metabolic index (Rg) was calculated as previously described (53).

Body composition analysis. Body composition was analyzed using proton TD-NMR signals on a body composition analyzer (Brucker Minispec). 
Indirect calorimetry. Indirect calorimetry was performed at the Comprehensive Metabolic Core at Northwestern University. Briefly, mice after 15 weeks of HFD were put into the TSE LabMaster Automated Phenotyping system. The system was maintained at a 12-hour dark, 12-hour light cycle, and mice had ad libitum access to water and food. Following a 3-day acclimation period, metabolic data were recorded over the next 4-day period, and data recorded during the light and dark phase were analyzed separately.

Measurement of TG content and NEFA. For liver and serum TG measurements, the Triglyceride Quantification kit (Biovision) or L type Triglyceride M reagent (Wako) was used. Livers and serum were collected at 9:00 a.m. under fed or 16-hour fasting conditions. Measured values were normalized to protein concentration in the lysate (Biovision) or liver wet weight prior to homogenization (Wako). For serum NEFA measurement, the Wako NEFA kit was used. Serum was collected at 9:00 a.m. under fed or 16-hour fasting conditions.

Serum FGF21 levels. For measurement of serum FGF21 levels, the R\&D mouse/rat FGF21 ELISA kit was used. Serum was collected at 9:00 a.m. after 16-hour fasting conditions unless otherwise indicated.

Evolutionary constraint sequence analysis. Constrained elements in multiple alignments were quantified using the Genomic Evolutionary Rate Profiling (GERP) score (54). The chromosomal location of the ARE sequence was obtained from AREsite and then entered into UCSC Genome Browser to obtain the GERP score for mammalian alignments. Scores were calculated as the average value across the indicated ARE site.

$R N A$ stability. Primary hepatocytes were plated at $\sim 80 \%$ confluence and were serum starved overnight $(0.1 \%$ FBS). The next morning, cells were serum stimulated (10\% FBS) for 1 hour to maximize TTP levels, followed by incubation in complete media with $7.5 \mu \mathrm{M}$ actinomycin D (MilliporeSigma) for up to 3 hours. Actinomycin D is a transcriptional inhibitor; thus, RNA levels reduce progressively following addition of this compound due to mRNA degradation. The rate of mRNA decay allows for determination of mRNA stability (14). No drop in cell viability was observed at the end of the treatment. RNA was collected and mRNA levels were analyzed by qPCR as described below.

$R N A$ co-IP. RNA co-IP was performed as previously described (14). Briefly, HepG2 cells were grown in complete media, collected, resuspended in Buffer A (10 mM Tris- $\mathrm{HCl}, \mathrm{pH} 7.6,1 \mathrm{mM} \mathrm{KAc}, 1.5 \mathrm{mM}$ MgAc, 2 mM DTT [MilliporeSigma], $10 \mu 1 / \mathrm{ml}$ ProteaseArrest inhibitors [G-Biosciences]), and lysed using Power Gen 500 homogenizer (Thermo Fisher Scientific), followed by centrifugation at 12,000 $\mathrm{g}$ for $10 \mathrm{~min}$ utes at $4^{\circ} \mathrm{C}$ to remove debris. Protein G Sepharose Fast Flow beads (MilliporeSigma) were incubated with human TTP or IgG antibody in the IP Buffer (10 mM Tris-HCl, pH 7.6, $1.5 \mathrm{mM} \mathrm{MgCl}$, $100 \mathrm{mM} \mathrm{NaCl}$, $0.5 \%$ Triton X-100 [MilliporeSigma], $10 \mu 1 / \mathrm{ml}$ ProteaseArrest inhibitors) at $4^{\circ} \mathrm{C}$ with continuous rotation for 4 hours. Beads were then washed 3-6 times with cold IP buffer, followed by incubation with HepG2 lysate at $4^{\circ} \mathrm{C}$ with continuous rotation for 2 hours, 6 washes with cold IP buffer to remove unbound RNA, and RNA collection as described above. Equal amounts of RNA were amplified by qPCR using 2 pairs of primers targeting FGF21, as well as the primers designed to target the regions near the $3^{\prime}$-UTR of known targets of TTP (VEGFA) and negative control genes (HPRT1). Expression of each gene was normalized to that of $18 S$ or $\beta$-actin, and data were expressed as fold enrichment over IgG control.

Luciferase assay. 3'-UTRs of human and mouse FGF21 were cloned into the pMIR-REPORT vector containing the gene for firefly luciferase (Ambion). WT MEFs were transfected with TTP as described above for 4 hours. Alternatively, HEK293 cells were transfected with WT TTP, C124R mutant TTP, or pEGFP N3 empty vector as described above for 4 hours. After transfection with TTP, firefly and Renilla luciferase constructs were transfected into the cells for another 4 hours, followed by incubation in complete medium for 16-20 hours. Cells were then harvested in passive lysis buffer (Promega). The luminescence was quantified using the Dual-Luciferase Reporter Assay System (Promega) on a luminometer (Turner Biosystems, Modulus Microplate) according to the manufacturer's protocol. Renilla luminescence was used as normalization control for variations in transfection efficiency.

Western blot. Livers were perfused with PBS flush via cardiac puncture prior to harvest. Approximately $10 \mathrm{mg}$ of perfused tissue was homogenized in RIPA buffer in the presence of ProteaseArrest protease inhibitors (G-Biosciences), centrifuged at 5,000 $\mathrm{g}$ for 15 minutes to remove debris, and protein concentration of the supernatant were determined by BCA assay (Invitrogen). Protein (50-100 $\mu \mathrm{g}$ ) was loaded on sodium dodecyl sulfate polyacrylamide gel electrophoresis gels and transferred to nitrocellulose membranes (Invitrogen). The membranes were probed with antibodies against TTP (William Rigby, Dartmouth University, Hanover, New Hampshire, USA), pAKT (Ser473) and AKT (Cell Signaling Technologies, 4060L and 
9072S, respectively), and GAPDH (Abcam, ab9485). Horseradish peroxidase-conjugated donkey anti-rabbit antibody were used as secondary antibodies (Jackson ImmunoResearch, 711-035-152) and visualized by SuperSignal Chemiluminescent substrates (Thermo Fisher Scientific). See complete unedited blots in the supplemental material.

$q P C R$. RNA was isolated with RNA STAT-60 (TEL-TEST Inc.), reverse transcribed with qScript cDNA SuperMix (Quanta), and amplified on a 7500 Fast Real-Time PCR system with PerfeCTa SYBR Green SuperMix (Quanta). Primers were designed using Primer3 (version 0.4.0) software to target sequences spanning an exon-intron-exon boundary, and their specificity was confirmed by running a dissociation curve. mRNA levels were calculated by the comparative Ct method and normalized to $A C T B, H P R T$, or $18 S$ rRNA. All primer sequences used in this manuscript are available upon request. Primer sequences are included in Supplemental Table 3.

Statistics. Data are expressed as mean \pm SEM. Statistical significance was assessed with the 2-tailed unpaired Student's $t$ test or 1-way ANOVA with post hoc Fisher's HSD test when appropriate; $P<0.05$ was considered statistically significant. The analyses were carried out using SPSS software (IBM).

Study approval. All animal studies performed at Northwestern University were approved by the IACUC at Northwestern University and were performed in accordance with guidelines from the NIH. All procedures required for the hyperinsulinemic-euglycemic clamp were approved by the Vanderbilt University Animal Care and Use Committee. For human tissue samples, informed consent was obtained from all biopsy patients. Protocols for human liver tissue procurement were approved by the IRB of Northwestern University.

\section{Author contributions}

KTS, MB, and HA designed the research. KTS, HCC, JSS, and ADJ performed the experiments and data analysis. PJB and JAW provided tools and reagents. BNF provided intellectual input. KTS wrote the manuscript, which all authors commented on. HA supervised the project.

\section{Acknowledgments}

The authors are grateful to all members of the FCVRI for their insightful comments and support. We thank Margrit Urbanek (Northwestern University) for procurement of human liver tissue biopsy samples, Navdeep Chandel (Northwestern University) for $A k t 1 / 2-\mathrm{KO}$ MEFs, and Roger Davis (University of Massachusetts) for providing the Fgf21 shRNA construct. Hyperinsulinemic-euglycemic clamps were performed by the Vanderbilt Mouse Metabolic Phenotyping Center (DK059637). The Vanderbilt Hormone Assay and Analytical Core performed the insulin analysis (DK059637 and DK020593). Indirect calorimetry was performed by the Comprehensive Metabolic Core at Northwestern University. HA is supported by the NIH (R01 HL127646, HL140973, and HL138982). KTS is supported by the NIH (F30DK102341 and T32GM008152). HCC is supported by the NIH (T32GM008152). JSS is supported by the NIH (F30DK109608 and T32GM008152). ADJ is supported by the NIH (F31HL132552 and T32GM008152). BNF is supported by the NIH (R01 DK078187). JAW is supported by the NIH (K08DK101757).

Address correspondence to: Hossein Ardehali, Tarry 14-733, 303 E Chicago Avenue Chicago, Illinois 60611, USA. Phone: 312.503.2342; Email: h-ardehali@northwestern.edu.

1. Badman MK, Pissios P, Kennedy AR, Koukos G, Flier JS, Maratos-Flier E. Hepatic fibroblast growth factor 21 is regulated by PPARalpha and is a key mediator of hepatic lipid metabolism in ketotic states. Cell Metab. 2007;5(6):426-437.

2. Ding X, et al. $\beta$ Klotho is required for fibroblast growth factor 21 effects on growth and metabolism. Cell Metab. 2012;16(3):387-393

3. Véniant MM, et al. FGF21 promotes metabolic homeostasis via white adipose and leptin in mice. PLoS ONE. $2012 ; 7(7):$ e40164.

4. Kharitonenkov A, et al. FGF-21 as a novel metabolic regulator. J Clin Invest. 2005;115(6):1627-1635.

5. Chen W, et al. Growth hormone induces hepatic production of fibroblast growth factor 21 through a mechanism dependent on lipolysis in adipocytes. J Biol Chem. 2011;286(40):34559-34566.

6. Chau MD, Gao J, Yang Q, Wu Z, Gromada J. Fibroblast growth factor 21 regulates energy metabolism by activating the AMPK-SIRT1-PGC-1alpha pathway. Proc Natl Acad Sci USA. 2010;107(28):12553-12558.

7. Xu J, et al. Fibroblast growth factor 21 reverses hepatic steatosis, increases energy expenditure, and improves insulin sensitivity in diet-induced obese mice. Diabetes. 2009;58(1):250-259

8. Castello A, Hentze MW, Preiss T. Metabolic Enzymes Enjoying New Partnerships as RNA-Binding Proteins. Trends Endocrinol Metab. 2015;26(12):746-757.

9. Blackshear PJ. Tristetraprolin and other $\mathrm{CCCH}$ tandem zinc-finger proteins in the regulation of mRNA turnover. Biochem Soc Trans. 2002;30(Pt 6):945-952. 
10. Fabian MR, et al. Structural basis for the recruitment of the human CCR4-NOT deadenylase complex by tristetraprolin. Nat Struct Mol Biol. 2013;20(6):735-739.

11. Lai WS, Carballo E, Strum JR, Kennington EA, Phillips RS, Blackshear PJ. Evidence that tristetraprolin binds to AU-rich elements and promotes the deadenylation and destabilization of tumor necrosis factor alpha mRNA. Mol Cell Biol. 1999;19(6):4311-4323.

12. Carballo E, Lai WS, Blackshear PJ. Feedback inhibition of macrophage tumor necrosis factor-alpha production by tristetraprolin. Science. 1998;281(5379):1001-1005.

13. Rounbehler RJ, et al. Tristetraprolin impairs myc-induced lymphoma and abolishes the malignant state. Cell. 2012;150(3):563-574.

14. Bayeva M, et al. mTOR regulates cellular iron homeostasis through tristetraprolin. Cell Metab. 2012;16(5):645-657.

15. Bouchard L, et al. ZFP36: a promising candidate gene for obesity-related metabolic complications identified by converging genomics. Obes Surg. 2007;17(3):372-382.

16. Lai WS, Stumpo DJ, Blackshear PJ. Rapid insulin-stimulated accumulation of an mRNA encoding a proline-rich protein. $J$ Biol Chem. 1990;265(27):16556-16563.

17. Cao H, Urban JF, Anderson RA. Insulin increases tristetraprolin and decreases VEGF gene expression in mouse 3T3-L1 adipocytes. Obesity (Silver Spring). 2008;16(6):1208-1218.

18. Bouchard L, Vohl MC, Deshaies Y, Rhéaume C, Daris M, Tchernof A. Visceral adipose tissue zinc finger protein 36 mRNA levels are correlated with insulin, insulin resistance index, and adiponectinemia in women. Eur J Endocrinol. 2007;157(4):451-457.

19. Moller DE. Potential role of TNF-alpha in the pathogenesis of insulin resistance and type 2 diabetes. Trends Endocrinol Metab. 2000;11(6):212-217.

20. Puig S, Vergara SV, Thiele DJ. Cooperation of two mRNA-binding proteins drives metabolic adaptation to iron deficiency. Cell Metab. 2008;7(6):555-564.

21. Puig S, Askeland E, Thiele DJ. Coordinated remodeling of cellular metabolism during iron deficiency through targeted mRNA degradation. Cell. 2005;120(1):99-110.

22. Mukherjee A, et al. FSTL3 deletion reveals roles for TGF-beta family ligands in glucose and fat homeostasis in adults. Proc Natl Acad Sci USA. 2007;104(4):1348-1353.

23. Emanuelli B, et al. Interplay between FGF21 and insulin action in the liver regulates metabolism. J Clin Invest. 2014;124(2):515-527.

24. Andersen B, Omar BA, Rakipovski G, Raun K, Ahrén B. Fibroblast growth factor 21 prevents glycemic deterioration in insulin deficient mouse models of diabetes. Eur J Pharmacol. 2015;764:189-194.

25. Fisher FM, Maratos-Flier E. Understanding the Physiology of FGF21. Annu Rev Physiol. 2016;78:223-241.

26. Lin Z, et al. Adiponectin mediates the metabolic effects of FGF21 on glucose homeostasis and insulin sensitivity in mice. Cell Metab. 2013;17(5):779-789.

27. Ge X, Chen C, Hui X, Wang Y, Lam KS, Xu A. Fibroblast growth factor 21 induces glucose transporter-1 expression through activation of the serum response factor/Ets-like protein-1 in adipocytes. J Biol Chem. 2011;286(40):34533-34541.

28. Mashili FL, et al. Direct effects of FGF21 on glucose uptake in human skeletal muscle: implications for type 2 diabetes and obesity. Diabetes Metab Res Rev. 2011;27(3):286-297.

29. Gaich G, et al. The effects of LY2405319, an FGF21 analog, in obese human subjects with type 2 diabetes. Cell Metab. 2013;18(3):333-340.

30. Fisher FM, et al. Obesity is a fibroblast growth factor 21 (FGF21)-resistant state. Diabetes. 2010;59(11):2781-2789.

31. Potthoff MJ, et al. FGF21 induces PGC-1alpha and regulates carbohydrate and fatty acid metabolism during the adaptive starvation response. Proc Natl Acad Sci USA. 2009;106(26):10853-10858.

32. De Sousa-Coelho AL, et al. FGF21 mediates the lipid metabolism response to amino acid starvation. J Lipid Res. 2013;54(7):1786-1797.

33. Tanaka N, et al. Role of fibroblast growth factor 21 in the early stage of NASH induced by methionine- and choline-deficient diet. Biochim Biophys Acta. 2015;1852(7):1242-1252.

34. So WY, Cheng Q, Xu A, Lam KS, Leung PS. Loss of fibroblast growth factor 21 action induces insulin resistance, pancreatic islet hyperplasia and dysfunction in mice. Cell Death Dis. 2015;6:e1707.

35. Li H, et al. Fibroblast growth factor 21 increases insulin sensitivity through specific expansion of subcutaneous fat. Nat Commun. 2018;9(1):272.

36. Fisher FM, et al. FGF21 regulates PGC-1 $\alpha$ and browning of white adipose tissues in adaptive thermogenesis. Genes Dev. 2012;26(3):271-281.

37. Pyrżak B, Demkow U, Kucharska AM. Brown Adipose Tissue and Browning Agents: Irisin and FGF21 in the Development of Obesity in Children and Adolescents. In: Pokorski M, ed. Noncommunicable Diseases. Cham, Switzerland: Springer International Publishing; 2015:25-34.

38. Hanssen MJ, et al. Serum FGF21 levels are associated with brown adipose tissue activity in humans. Sci Rep. 2015;5:10275.

39. Zhang Y, et al. The starvation hormone, fibroblast growth factor-21, extends lifespan in mice. Elife. 2012;1:e00065.

40. Ge X, Wang Y, Lam KSL, Xu A. Metabolic actions of FGF21: molecular mechanisms and therapeutic implications. Acta Pharmaceutica Sinica B. 2012;2(4):350-357.

41. Coskun T, et al. Fibroblast growth factor 21 corrects obesity in mice. Endocrinology. 2008;149(12):6018-6027.

42. BonDurant LD, et al. FGF21 Regulates Metabolism Through Adipose-Dependent and -Independent Mechanisms. Cell Metab. 2017;25(4):935-944.e4.

43. Emanuelli B, et al. Interplay between FGF21 and insulin action in the liver regulates metabolism. J Clin Invest. $2015 ; 125(1): 458$.

44. Brewer BY, Malicka J, Blackshear PJ, Wilson GM. RNA sequence elements required for high affinity binding by the zinc finger domain of tristetraprolin: conformational changes coupled to the bipartite nature of Au-rich MRNA-destabilizing motifs. $J$ Biol Chem. 2004;279(27):27870-27877.

45. Hudson BP, Martinez-Yamout MA, Dyson HJ, Wright PE. Recognition of the mRNA AU-rich element by the zinc finger domain of TIS11d. Nat Struct Mol Biol. 2004;11(3):257-264.

46. Pettersson US, Waldén TB, Carlsson PO, Jansson L, Phillipson M. Female mice are protected against high-fat diet induced metabolic syndrome and increase the regulatory T cell population in adipose tissue. PLoS One. 2012;7(9):e46057. 
47. Uhlén M, et al. Proteomics. Tissue-based map of the human proteome. Science. 2015;347(6220):1260419.

48. Fallmann J, Sedlyarov V, Tanzer A, Kovarik P, Hofacker IL. AREsite2: an enhanced database for the comprehensive investigation of AU/GU/U-rich elements. Nucleic Acids Res. 2016;44(D1):D90-D95.

49. Berglund ED, et al. Glucose metabolism in vivo in four commonly used inbred mouse strains. Diabetes. 2008;57(7):1790-1799.

50. Ayala JE, Bracy DP, McGuinness OP, Wasserman DH. Considerations in the design of hyperinsulinemic-euglycemic clamps in the conscious mouse. Diabetes. 2006;55(2):390-397.

51. Finegood DT, Bergman RN, Vranic M. Estimation of endogenous glucose production during hyperinsulinemic-euglycemic glucose clamps. Comparison of unlabeled and labeled exogenous glucose infusates. Diabetes. 1987;36(8):914-924.

52. Steele R, Wall JS, De Bodo RC, Altszuler N. Measurement of size and turnover rate of body glucose pool by the isotope dilution method. Am J Physiol. 1956;187(1):15-24.

53. Kraegen EW, James DE, Jenkins AB, Chisholm DJ. Dose-response curves for in vivo insulin sensitivity in individual tissues in rats. Am J Physiol. 1985;248(3 Pt 1):E353-E362.

54. Davydov EV, Goode DL, Sirota M, Cooper GM, Sidow A, Batzoglou S. Identifying a high fraction of the human genome to be under selective constraint using GERP++. PLoS Comput Biol. 2010;6(12):e1001025. 